\title{
THE NEWTON TREE: GEOMETRIC INTERPRETATION AND APPLICATIONS TO THE MOTIVIC ZETA FUNCTION AND THE LOG CANONICAL THRESHOLD
}

\author{
PIERRETTE CASSOU-NOGUÈS AND WILLEM VEYS
}

\begin{abstract}
Let $\mathcal{I}$ be an arbitrary ideal in $\mathbb{C}[[x, y]]$. We use the Newton algorithm to compute by induction the motivic zeta function of the ideal, yielding only few poles, associated to the faces of the successive Newton polygons. We associate a minimal Newton tree to $\mathcal{I}$, related to using good coordinates in the Newton algorithm, and show that it has a conceptual geometric interpretation in terms of the $\log$ canonical model of $\mathcal{I}$. We also compute the log canonical threshold from a Newton polygon and strengthen Corti's inequalities.
\end{abstract}

\section{INTRODUCTION}

We introduced in [7] the Newton algorithm as an efficient way to study an arbitrary ideal $\mathcal{I}$ in $\mathbb{C}[[x, y]]$, involving a finite succession of Newton polygons. We showed for instance how to compute its integral closure and the Hilbert-Samuel multiplicity of $\mathcal{I}$ in a nice combinatorial way. In this article we provide more applications. First we show how the Newton algorithm can be used to compute by induction the motivic zeta function of $\mathcal{I}$, yielding candidate poles associated to the faces of the successive Newton polygons (Theorem 3.4). This method is much more efficient than other ones in the literature, and almost all candidate poles are true poles. The classical method uses a $\log$ principalization of $\mathcal{I}$ and yields generally an enormous amount of false candidate poles. On the other hand the formula in [19, Theorem 6.1] has only the actual poles, but it involves horrible determinants, that are in fact again expressed in terms of a log principalization.

In 7 ] we moreover codified most of the data of the algorithm in a useful combinatorial object, the Newton tree of $\mathcal{I}$. This (decorated) tree depends heavily on the chosen resolution. We introduce an operator on Newton trees, exchange of vertical edge, and show that it corresponds to a change of coordinates (Proposition 4.5). We define the notion of minimal Newton tree, we prove that any Newton tree can be converted into a minimal one by exchanging vertical edges (Proposition 4.3), and that this is related to the use of so-called (very) good coordinates. Coming back to the motivic zeta function, some very particular faces do not give poles (Proposition 3.5), and these faces will not appear when one uses very good coordinates.

A minimal Newton tree of $\mathcal{I}$ has also a conceptual geometric interpretation. Roughly its vertices correspond to the exceptional components in the log canonical model of the ideal, and its edges to intersections of components (Theorem 5.1). Also the decorations of the Newton tree correspond exactly to the 'classical' decorations on a dual tree of a curve configuration. For vertex decorations this was shown in [6] in the context of principal ideals; the argument is still valid for arbitrary ideals. For edge decorations the result is new (Proposition 5.2).

We also show a generalization of a result known in the case where the ideal is principal [10] [3], that is, that there exists a system of coordinates such that the log canonical threshold

First author is partially supported by the grants MTM2013-45710-C02-01-P and MTM2013-45710 -C0202-P. Second author is partially supported by the KU Leuven grant OT/11/069. 
can be computed from the Newton polygon (Theorem 6.5). In [8] and [4 the authors prove inequalities between the Hilbert-Samuel multiplicity of an ideal and its log canonical threshold. Using the computations via Newton polygons these inequalities appear to be simple geometric facts (Corollary 6.14), and using the computations via the Newton algorithm they can be strengthened (Theorem 6.16).

The article is organized as follows. In the next section we recall the Newton algorithm for ideals. In section 3 we apply the Newton algorithm to the computation of the motivic zeta function. In section 4, after recalling the construction of Newton trees, we compare them in different systems of coordinates and we introduce the notion of minimal Newton tree. In section 5 we show that the dual tree of the log canonical model of the ideal can be deduced from a minimal Newton tree. Finally, we study the log canonical threshold and the inequalities involving the Hilbert-Samuel multiplicity in section 6 .

\section{NeWTON Algorithm FOR AN IDEAL}

We recall briefly the essential definitions and results from [7] concerning the Newton algorithm.

2.1. Newton polygon. For any set $E \subset \mathbb{N} \times \mathbb{N}$, denote by $\Delta(E)$ the smallest convex set containing $E+\mathbb{R}_{+}^{2}=\left\{a+b \mid a \in E, b \in \mathbb{R}_{+}^{2}\right\}$. A set $\Delta \subset \mathbb{R}^{2}$ is a Newton diagram if there exists a set $E \subset \mathbb{N} \times \mathbb{N}$ such that $\Delta=\Delta(E)$. The smallest set $E_{0} \subset \mathbb{N} \times \mathbb{N}$ such that $\Delta=\Delta\left(E_{0}\right)$ is called the set of vertices of a Newton diagram $\Delta$; it is a finite set. Let $E_{0}=\left\{v_{0}, \cdots, v_{m}\right\}$, with $v_{i}=\left(\alpha_{i}, \beta_{i}\right) \in \mathbb{N} \times \mathbb{N}$ for $i=0, \cdots, m$, and $\alpha_{i-1}<\alpha_{i}, \beta_{i-1}>\beta_{i}$ for $i=1, \cdots, m$.

For $i \in\{1, \cdots, m\}$, denote $S_{i}=\left[v_{i-1}, v_{i}\right]$ and by $l_{S_{i}}$ the line supporting the segment $S_{i}$. We call $\mathcal{N}(\Delta)=\cup_{1 \leq i \leq m} S_{i}$ the Newton polygon of $\Delta$ and the $S_{i}$ its faces. The Newton polygon $\mathcal{N}(\Delta)$ is empty if and only if $E_{0}=\left\{v_{0}=\left(\alpha_{0}, \beta_{0}\right)\right\}$. The Newton diagram $\Delta$ has also two non-compact faces: the vertical half-line starting at $v_{0}$, and the horizontal half-line starting at $v_{m}$. The integer $h(\Delta)=\beta_{0}-\beta_{m}$ is called the height of $\Delta$. Let

$$
f(x, y)=\sum_{(\alpha, \beta) \in \mathbb{N} \times \mathbb{N}} c_{\alpha, \beta} x^{\alpha} y^{\beta} \in \mathbb{C}[[x, y]] .
$$

We define the support of $f$ as

$$
\operatorname{Supp} f=\left\{(\alpha, \beta) \in \mathbb{N} \times \mathbb{N} \mid c_{\alpha, \beta} \neq 0\right\} .
$$

We denote $\Delta(f)=\Delta(\operatorname{Supp} f)$ and $\mathcal{N}(f)=\mathcal{N}(\Delta(f))$. Let $l$ be a line in $\mathbb{R}^{2}$. We define the initial part of $f$ with respect to $l$ as

$$
\operatorname{in}(f, l)=\sum_{(\alpha, \beta) \in l} c_{\alpha, \beta} x^{\alpha} y^{\beta}
$$

If the line $l$ has equation $p \alpha+q \beta=N$, with $(p, q) \in\left(\mathbb{N}^{*}\right)^{2}$ and $\operatorname{gcd}(p, q)=1$, then $\operatorname{in}(f, l)$ is zero or a monomial or, if $l=l_{S}$ for some segment $S$ of $\mathcal{N}(\Delta)$, of the form

$$
\operatorname{in}(f, l)=x^{a_{l}} y^{b_{l}} F_{S}\left(x^{q}, y^{p}\right),
$$

where $\left(a_{l}, b_{l}\right) \in \mathbb{N}^{2}$ and

$$
F_{S}(x, y)=c \prod_{1 \leq i \leq n}\left(y-\mu_{i} x\right)^{\nu_{i}}
$$

with $c \in \mathbb{C}^{*}, n \in \mathbb{N}^{*}, \mu_{i} \in \mathbb{C}^{*}$ (all different) and $\nu_{i} \in \mathbb{N}^{*}$. 
THE NEWTON TREE: GEOMETRIC INTERPRETATION AND APPLICATIONS

Now let $\mathcal{I}=\left(f_{1}, \cdots, f_{r}\right)$ be a non-trivial ideal in $\mathbb{C}[[x, y]]$. We define

$$
\Delta(\mathcal{I})=\Delta\left(\cup_{1 \leq i \leq r} \operatorname{Supp} f_{i}\right) \quad \text { and } \quad \mathcal{N}(\mathcal{I})=\mathcal{N}(\Delta(\mathcal{I}))
$$

When $\mathcal{I}=(f)$ we simply write $\Delta(f)$ and $\mathcal{N}(f)$. For a segment $S$ of $\mathcal{N}(\mathcal{I})$ we denote by $\operatorname{in}(\mathcal{I}, S)$ the ideal generated by the $\operatorname{in}\left(f_{i}, l_{S}\right), 1 \leq i \leq r$, and call it the initial ideal of $\mathcal{I}$ with respect to $S$.

One easily verifies that $\Delta(\mathcal{I})=\Delta\left(\cup_{f \in \mathcal{I}} \operatorname{Supp} f\right)$; hence the sets $\Delta(\mathcal{I})$ and $\mathcal{N}(\mathcal{I})$ and the ideals $\operatorname{in}(\mathcal{I}, S)$ depend only on $\mathcal{I}$, not on a system of generators of $\mathcal{I}$.

Let $S$ be a face of $\mathcal{N}(\mathcal{I})$ and $p_{S} \alpha+q_{S} \beta=N_{S}$ be the equation of $l_{S}$, with $\operatorname{gcd}\left(p_{S}, q_{S}\right)=1$ as before. Then $\operatorname{in}(\mathcal{I}, S)$ is of the form

$$
\operatorname{in}(\mathcal{I}, S)=\left(x^{a_{S}} y^{b_{S}} F_{\mathcal{I}, S}\left(x^{q_{S}}, y^{p_{S}}\right)\right)
$$

or

$$
\operatorname{in}(\mathcal{I}, S)=x^{a_{S}} y^{b_{S}} F_{\mathcal{I}, S}\left(x^{q_{S}}, y^{p_{S}}\right)\left(k_{1}\left(x^{q_{S}}, y^{p_{S}}\right), \cdots, k_{s}\left(x^{q_{S}}, y^{p_{S}}\right)\right)
$$

with $s \geq 2$, where $F_{\mathcal{I}, S}, k_{1}, \cdots, k_{s}$ are homogeneous polynomials, $F_{\mathcal{I}, S}$ is not divisible by $x$ or $y$ and $k_{1}, \cdots, k_{s}$ are coprime and of the same degree $d_{S}$. In the first case we put $d_{S}=0$. The polynomial $F_{\mathcal{I}, S}$, monic in $y$, is called the face polynomial (it can be identically one). A face $S$ is called a dicritical face if $\operatorname{in}(\mathcal{I}, S)$ is not a principal ideal. Thus it is dicritical if and only if $d_{S} \geq 1$.

In the sequel we will also call the equation of the supporting line of a face simply the equation of the face.

\subsection{Newton maps.}

Definition 2.1. Let $(p, q) \in\left(\mathbb{N}^{*}\right)^{2}$ with $\operatorname{gcd}(p, q)=1$. Take $\left(p^{\prime}, q^{\prime}\right) \in \mathbb{N}^{2}$ such that $p p^{\prime}-q q^{\prime}=$ 1. Let $\mu \in \mathbb{C}^{*}$. Define

$$
\begin{array}{rccc}
\sigma_{(p, q, \mu)}: & \mathbb{C}[[x, y]] & \longrightarrow & \mathbb{C}\left[\left[x_{1}, y_{1}\right]\right] \\
& f(x, y) & \mapsto & f\left(\mu^{q^{\prime}} x_{1}^{p}, x_{1}^{q}\left(y_{1}+\mu^{p^{\prime}}\right)\right) .
\end{array}
$$

We say that the map $\sigma_{(p, q, \mu)}$ is a Newton map.

The numbers $\left(p^{\prime}, q^{\prime}\right)$ are introduced only to avoid taking roots of complex numbers. In the sequel we will always assume that $p^{\prime} \leq q$ and $q^{\prime}<p$. This will make procedures canonical.

Let $\mathcal{I}=\left(f_{1}, \cdots, f_{r}\right)$ be a non-trivial ideal in $\mathbb{C}[[x, y]]$. Let $\sigma_{(p, q, \mu)}$ be a Newton map. We denote by $\sigma_{(p, q, \mu)}(\mathcal{I})$ the ideal in $\mathbb{C}\left[\left[x_{1}, y_{1}\right]\right]$ generated by the $\sigma_{(p, q, \mu)}\left(f_{i}\right)$ for $i=1, \cdots, r$. Since a Newton map is a ring homomorphism, this ideal does not depend on the choice of the generators of $\mathcal{I}$.

Lemma 2.2. [7, Lemma 2.9] Let $\mathcal{I}$ be a non-trivial ideal in $\mathbb{C}[[x, y]]$ and $\sigma_{(p, q, \mu)}(\mathcal{I})=\mathcal{I}_{1}$.

(1) If there does not exist a face $S$ of $\mathcal{N}(\mathcal{I})$ whose supporting line has equation $p \alpha+q \beta=N$ with $N \in \mathbb{N}$, then the ideal $\mathcal{I}_{1}$ is principal, generated by a power of $x_{1}$.

(2) If there exists a face $S$ of $\mathcal{N}(\mathcal{I})$ whose supporting line has equation $p \alpha+q \beta=N_{0}$ for some $N_{0} \in \mathbb{N}$, and if $F_{\mathcal{I}, S}(1, \mu) \neq 0$, then $\mathcal{I}_{1}=\left(x_{1}^{N_{0}}\right)$.

(3) If there exists a face $S$ of $\mathcal{N}(\mathcal{I})$ whose supporting line has equation $p \alpha+q \beta=N_{0}$ for some $N_{0} \in \mathbb{N}$, and if $F_{\mathcal{I}, S}(1, \mu)=0$, then $\mathcal{I}_{1}=\left(x_{1}^{N_{0}}\right) \mathcal{I}_{1}^{\prime}$ and the height of the Newton polygon of $\mathcal{I}_{1}$ is less than or equal to the multiplicity of $\mu$ as root of $F_{\mathcal{I}, S}(1, X)$, 
2.3. Newton algorithm. Given an ideal $\mathcal{I}$ in $\mathbb{C}[[x, y]]$ and a Newton map $\sigma_{(p, q, \mu)}$, we denote by $\mathcal{I}_{\sigma}$ the ideal $\sigma_{(p, q, \mu)}(\mathcal{I})$. Consider a sequence $\Sigma_{n}=\left(\sigma_{1}, \cdots, \sigma_{n}\right)$ of length $n$ of Newton maps. We define $\mathcal{I}_{\Sigma_{n}}$ by induction:

$$
\mathcal{I}_{\Sigma_{1}}=\mathcal{I}_{\sigma_{1}}, \cdots, \mathcal{I}_{\Sigma_{i}}=\left(\mathcal{I}_{\Sigma_{i-1}}\right)_{\sigma_{i}}, \cdots, \mathcal{I}_{\Sigma_{n}}=\left(\mathcal{I}_{\Sigma_{n-1}}\right)_{\sigma_{n}}
$$

Theorem 2.3. [7, Theorem 2.10] Let $\mathcal{I}$ be a non-trivial ideal in $\mathbb{C}[[x, y]]$. There exists an integer $n_{0}$ such that, for any sequence $\Sigma_{n}=\left(\sigma_{1}, \cdots, \sigma_{n}\right)$ of Newton maps of length at least $n_{0}$, the ideal $\mathcal{I}_{\Sigma_{n}}$ is principal, generated by $x^{k}(y+h(x))^{\nu}$ with $h \in x \mathbb{C}[[x]]$ and $(k, \nu) \in \mathbb{N} \times \mathbb{N}$.

Example 1. We consider in $\mathbb{C}[[x, y]]$ the ideal

$$
\mathcal{I}=\left(y^{4}(y+x)\left(y^{2}-3 x\right),\left((y+x)^{3}+x^{8}\right)\left(y^{2}-3 x\right)\right) .
$$

Its Newton polygon is given in Figure 1.

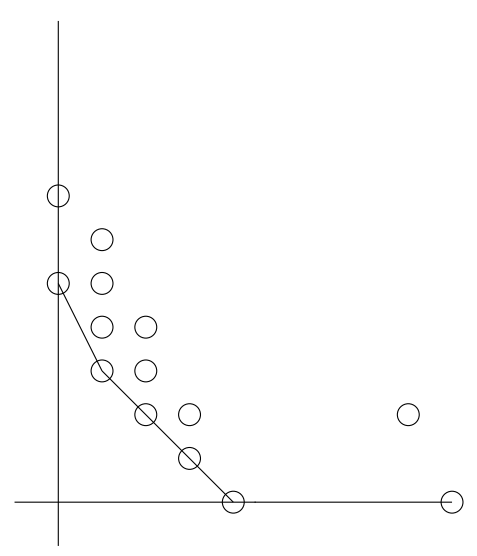

FIGURE 1.

The faces $S_{1}$ and $S_{2}$ have supporting lines with equations $2 \alpha+\beta=5$ and $\alpha+\beta=4$, respectively. The initial ideals of $\mathcal{I}$ with respect to these segments are

$$
\operatorname{in}\left(\mathcal{I}, S_{1}\right)=\left(y^{3}\left(y^{2}-3 x\right)\right) \quad \text { and } \quad \operatorname{in}\left(\mathcal{I}, S_{2}\right)=\left(x(y+x)^{3}\right) .
$$

Both segments are not dicritical; their face polynomials are $F_{\mathcal{I}, S_{1}}=y^{2}-3 x$ and $F_{\mathcal{I}, S_{2}}=$ $(y+x)^{3}$, respectively. We first consider the Newton map $\sigma_{(p, q, \mu)}=\sigma_{(2,1,3)}$ associated to $S_{1}$ and $\mu=3$. It is given by the substitution

$$
x=3 x_{1}^{2}, \quad y=x_{1}\left(y_{1}+3\right) .
$$

The image ideal $\mathcal{I}_{1}$ is given by

$$
\begin{aligned}
\mathcal{I}_{1}= & \left(x_{1}^{4}\left(y_{1}+3\right)^{4}\left(x_{1} y_{1}+3 x_{1}+3 x_{1}^{2}\right)\left(x_{1}^{2}\left(y_{1}+3\right)^{2}-9 x_{1}^{2}\right),\right. \\
& \left.\quad\left(\left(x_{1} y_{1}+3 x_{1}+3 x_{1}^{2}\right)^{3}+3^{8} x_{1}^{16}\right)\left(x_{1}^{2}\left(y_{1}+3\right)^{2}-9 x_{1}^{2}\right)\right) \\
= & \left(x_{1}^{7}\left(y_{1}^{2}+6 y_{1}\right), x_{1}^{5}\left(y_{1}^{2}+6 y_{1}\right)\right) \\
= & \left(x_{1}^{5} y_{1}\right) .
\end{aligned}
$$

It is a monomial ideal, hence we stop the procedure for $S_{1}$.

Next we consider the Newton map $\sigma_{(p, q, \mu)}=\sigma_{(1,1,-1)}$ associated to $S_{2}$ and $\mu=-1$. It is given by the substitution

$$
x=x_{1}, \quad y=x_{1}\left(y_{1}-1\right) .
$$


The image ideal $\mathcal{I}_{1}$ is given by

$$
\begin{aligned}
\mathcal{I}_{1} & =\left(x_{1}^{4}\left(y_{1}-1\right)^{4} x_{1} y_{1}\left(x_{1}^{2}\left(y_{1}-1\right)^{2}-3 x_{1}\right),\left(x_{1}^{3} y_{1}^{3}+x_{1}^{8}\right)\left(x_{1}^{2}\left(y_{1}-1\right)^{2}-3 x_{1}\right)\right) \\
& =\left(x_{1}^{6} y_{1}, x_{1}^{4}\left(y_{1}^{3}+x_{1}^{5}\right)\right) \\
& =x_{1}^{4}\left(x_{1}^{2} y_{1}, y_{1}^{3}+x_{1}^{5}\right) .
\end{aligned}
$$

Its Newton polygon is given in Figure 2.

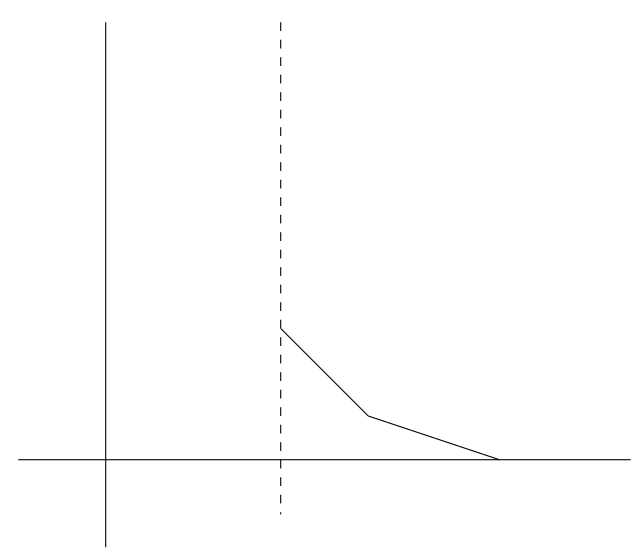

FiguRE 2.

The faces $S_{1}^{\prime}$ and $S_{2}^{\prime}$ have supporting lines with equations $\alpha+\beta=7$ and $\alpha+3 \beta=9$, respectively. The initial ideals of $\mathcal{I}_{1}$ with respect to these segments are

$$
\operatorname{in}\left(\mathcal{I}_{1}, S_{1}^{\prime}\right)=x_{1}^{4} y_{1}\left(x_{1}^{2}, y_{1}^{2}\right) \quad \text { and } \quad \operatorname{in}\left(\mathcal{I}_{1}, S_{2}^{\prime}\right)=x_{1}^{6}\left(y_{1}, x_{1}^{3}\right) .
$$

Both segments are dicritical and have constant face polynomial; their degrees are $d_{S_{1}^{\prime}}=2$ and $d_{S_{2}^{\prime}}=1$.

We continue with the Newton maps $\sigma_{(1,1, \mu)}$ associated to $S_{1}^{\prime}$ and $\sigma_{(1,3, \mu)}$ associated to $S_{2}^{\prime}$, where $\mu$ is arbitrary in $\mathbb{C}^{*}$. These lead to monomial ideals, hence we stop the procedure.

It should be clear that more generally, when a face $S$ of a Newton polygon is dicritical, the associated Newton map $\sigma_{(p, q, \mu)}$ induces a monomial ideal for all $\mu \in \mathbb{C}^{*}$ that are not roots of the face polynomial $F_{\mathcal{I}, S}$. Therefore it is not necessary to compute such Newton maps explicitly.

In [7] we introduced a notion of depth of an ideal: the least possible value of $n_{0}$ in Theorem 2.3. This can also be formulated as follows.

Definition 2.4. Let $\mathcal{I}$ be a non-trivial ideal in $\mathbb{C}[[x, y]]$. We define the depth of $\mathcal{I}$, denoted by $d(\mathcal{I})$, by induction. If $\mathcal{I}$ is principal, generated by $x^{k}(y+h(x))^{\nu}$ with $h \in x \mathbb{C}[[x]]$ and $(k, \nu) \in \mathbb{N} \times \mathbb{N}$, we say that its depth is 0 . Otherwise, we define

$$
d(\mathcal{I})=\max d\left(\mathcal{I}_{\sigma}\right)+1,
$$

where the maximum is taken over all possible Newton maps. 


\section{Computation of the Motivic zeta Function of AN IDEAL}

In this section we describe an efficient algorithm for the motivic zeta function of an ideal in $\mathbb{C}[[x, y]]$ in terms of the Newton algorithm. The method is the one used in [1][2] for quasi-ordinary hypersurface singularities.

First we introduce the ingredients needed to define the motivic zeta function.

Let $\mathcal{G}$ be the Grothendieck ring of algebraic varieties over $\mathbb{C}$. It is the abelian group generated by the symbols $[S]$, where $S$ is an algebraic variety over $\mathbb{C}$, with the relations $[S]=\left[S^{\prime}\right]$ when $S$ and $S^{\prime}$ are isomorphic, and

$$
[S]=\left[S \backslash S^{\prime}\right]+\left[S^{\prime}\right]
$$

when $S^{\prime}$ is closed in $S$. The product is defined by

$$
[S] \cdot\left[S^{\prime}\right]=\left[S \times S^{\prime}\right] .
$$

For later use we mention the product formula for bundles: if the variety $X$ is a locally trivial fibration over $B$ with fibre $F$, then $[X]=[B] \cdot[F]$ in $\mathcal{G}$.

We denote $\mathbb{L}=\left[\mathbb{A}_{\mathbb{C}}^{1}\right]$ and $\mathcal{G}_{\text {loc }}=\mathcal{G}\left[\mathbb{L}^{-1}\right]$.

We recall briefly the notions of jet scheme and arc scheme. A reference in the context of arbitrary schemes is [15]. The functor $\cdot \times_{\operatorname{Spec} \mathbb{C}} \operatorname{Spec} \mathbb{C}[t] /\left(t^{n+1}\right)$ on the category of $\mathbb{C}$-schemes has a right adjoint, denoted by $\mathcal{L}_{n}$. We call $\mathcal{L}_{n}(X)$ the $n$-jet scheme of $X$ and its closed points $n$-jets on $X$. For $m \geq n$, the closed immersions Spec $\mathbb{C}[t] /\left(t^{n+1}\right) \hookrightarrow \operatorname{Spec} \mathbb{C}[t] /\left(t^{m+1}\right)$, defined by reduction modulo $t^{n+1}$, induce canonical (projection) morphisms $\pi_{n}^{m}: \mathcal{L}_{m}(X) \rightarrow \mathcal{L}_{n}(X)$. These morphisms being affine, the projective limit

$$
\mathcal{L}(X):=\lim _{\longleftarrow} \mathcal{L}_{n}(X)
$$

exists as a $\mathbb{C}$-scheme; it is called the arc scheme of $X$ and its $\mathbb{C}$-points are called arcs on $X$. Denote by $\pi_{n}: \mathcal{L}(X) \longrightarrow \mathcal{L}_{n}(X)$ the natural projection morphisms.

From now on we take $X=\operatorname{Spec} \mathbb{C}[[x, y]]$ and we consider only those arcs and jets attached at the origin, that is, mapped by $\pi_{0}$ or $\pi_{0}^{n}$ to the origin in $X=\mathcal{L}_{0}(X)$. We denote these schemes (and also the sets of their $\mathbb{C}$-points) by $\mathcal{L}^{0}\left(\mathbb{C}^{2}\right)$ and $\mathcal{L}_{n}^{0}\left(\mathbb{C}^{2}\right)$, respectively. Note that they are isomorphic to Spec $\mathbb{C}\left[a_{i}, b_{i}\right]_{i \in \mathbb{N}^{*}}$ and Spec $\mathbb{C}\left[a_{1}, b_{1}, \ldots, a_{n}, b_{n}\right]$, respectively. For example an $n$-jet is denoted as $\left(a_{1} t+a_{2} t^{2}+\cdots+a_{n} t^{n}, b_{1} t+b_{2} t^{2}+\cdots+b_{n} t^{n}\right)$.

A cylinder in $\mathcal{L}^{0}\left(\mathbb{C}^{2}\right)$ is a subset of the form $A=\left(\pi_{n}\right)^{-1}(C)$, with $C$ a constructible subset of $\mathcal{L}_{n}^{0}\left(\mathbb{C}^{2}\right)$, for some $n \in \mathbb{N}$. For such a set $A$ we have that $\left[\pi_{m}(A)\right] \mathbb{L}^{-2 m}=\left[\pi_{n}(A)\right] \mathbb{L}^{-2 n}=$ $[C] \mathbb{L}^{-2 n}$ for all $m \geq n$, and one defines its motivic measure $\mu(A)$ as this value:

$$
\mu(A)=\left[\pi_{n}(A)\right] \mathbb{L}^{-2 n} \in \mathcal{G}_{\mathrm{loc}} .
$$

Let $\mathcal{I}$ be a non-trivial ideal in $\mathbb{C}[[x, y]]$. We write $\mathcal{I}=\left(x^{N}\right) \mathcal{I}^{\prime}$, where $\mathcal{I}^{\prime}$ is not divisible by $x$. Let $\omega$ be a regular differential 2 -form of the form

$$
\omega=x^{\nu-1} d x \wedge d y \text {. }
$$

We assume that if $N=0$, then $\nu=1$.

For $\phi \in \mathcal{L}^{0}\left(\mathbb{C}^{2}\right)$ we define

$$
\operatorname{ord}_{\phi} \mathcal{I}=\min \{\operatorname{ord}(f \circ \phi) \mid f \in \mathcal{I}\} .
$$

For $m \in \mathbb{N}$ and $n \in \mathbb{N}^{*}$ we consider

$$
V_{n, m}=\left\{\phi \in \mathcal{L}^{0}\left(\mathbb{C}^{2}\right) \mid \operatorname{ord}_{\phi} \mathcal{I}=n, \operatorname{ord}(\omega \circ \phi)=m\right\} .
$$


Note that the motivic measure of this collection of arcs is completely determined by the corresponding collection of $n$-jets

$$
\pi_{n}\left(V_{n, m}\right)=\left\{\phi \in \mathcal{L}_{n}^{0}\left(\mathbb{C}^{2}\right) \mid \operatorname{ord}_{\phi} \mathcal{I}=n, \operatorname{ord}(\omega \circ \phi)=m\right\}
$$

The motivic zeta function of $\mathcal{I}$ and $\omega$ is

$$
\zeta(\mathcal{I}, \omega)(T)=\sum_{n \geq 1}\left(\sum_{m \geq 0} \mu\left(V_{n, m}\right) \mathbb{L}^{-m}\right) T^{n} \in \mathcal{G}_{\text {loc }}[[T]] .
$$

The hypothesis on $\omega$ ensures that the sum over $m$ is finite.

The motivic zeta function was originally introduced for a principal ideal $\mathcal{I}$ (in a polynomial ring) [9]. The generalization to arbitrary ideals is straightforward (see for instance [21]). Incorporating also a differential form is natural in the process of studying or computing a motivic zeta function, see e.g. [2][20].

An important result in [9] is that motivic zeta functions are in fact rational functions (in $T$ ); this is proven by providing a formula in terms of a chosen embedded resolution of singularities. The proof extends to zeta functions associated to a general ideal and a differential form. However, in that formula generally a large amount of false candidate poles occur. In dimension 2 the results of [19] provide in principle a compact formula with only actual poles in the denominator, but the computation is a combinatorial disaster.

We now start deriving an efficient algorithmic way to compute $\zeta(\mathcal{I}, \omega)(T)$, based on the Newton algorithm, yielding in particular an 'almost minimal' denominator. (In the next section we explain a slight modification resulting in the optimal denominator.)

First we consider the case where the ideal $\mathcal{I}$ is principal, generated by a monomial:

$$
\mathcal{I}=\left(x^{N_{1}} y^{N_{2}}\right) \text {. }
$$

Let

$$
\phi(t)=\left\{\begin{array}{l}
x=c_{1} t^{k_{1}}+\cdots+a_{n} t^{n}+\cdots \\
y=c_{2} t^{k_{2}}+\cdots+b_{n} t^{n}+\cdots
\end{array}\right.
$$

be an arc in $V_{n, m}$, where $c_{1}, c_{2} \in \mathbb{C}^{*}$. We have

$$
\begin{gathered}
\operatorname{ord}_{\phi} \mathcal{I}=k_{1} N_{1}+k_{2} N_{2}=n, \\
\operatorname{ord}(\omega \circ \phi)=(\nu-1) k_{1}=m, \\
\mu\left(V_{n, m}\right)=\left[\pi_{n}\left(V_{n, m}\right)\right] \mathbb{L}^{-2 n} .
\end{gathered}
$$

Then

$$
\begin{aligned}
\zeta(\mathcal{I}, \omega)(T) & =(\mathbb{L}-1)^{2} \sum_{k_{1} \geq 1, k_{2} \geq 1} \mathbb{L}^{n-k_{1}} \mathbb{L}^{n-k_{2}} \mathbb{L}^{-2 n} \mathbb{L}^{-(\nu-1) k_{1}} T^{n} \\
& =(\mathbb{L}-1)^{2} \sum_{k_{1} \geq 1, k_{2} \geq 1} \mathbb{L}^{-\nu k_{1}-k_{2}} T^{k_{1} N_{1}+k_{2} N_{2}} \\
& =(\mathbb{L}-1)^{2} \frac{\mathbb{L}^{-\nu} T^{N_{1}}}{1-\mathbb{L}^{-\nu} T^{N_{1}}} \frac{\mathbb{L}^{-1} T^{N_{2}}}{1-\mathbb{L}^{-1} T^{N_{2}}} .
\end{aligned}
$$

Remark 3.1. Above we tacitly assume that $N_{1}$ or $N_{2}$ can be zero. In that case a factor of the form $1-\mathbb{L}^{-\nu}$ appears in the denominator of the formula. More generally, in the rest of this section, formulas for $\zeta(\mathcal{I}, \omega)(T)$ as a rational function in $T$ have coefficients in a further localization of $\mathcal{G}_{\text {loc }}$, namely with respect to the elements $1-\mathbb{L}^{-a}, a \in \mathbb{Z}^{*}$. This is standard in such computations. 
To compute the zeta function of an arbitrary ideal, we use induction on the depth of the ideal. If the ideal has depth 0, we already computed its zeta function (since it does not depend on the chosen coordinate system). For an ideal $\mathcal{I}$ of depth at least 1 , we derive a formula computing the zeta function of $\mathcal{I}$ in terms of the zeta functions of the ideals $\mathcal{I}_{\sigma}$. First we collect the necessary data and notation.

Definition 3.2. Let $\mathcal{I}$ be a non-trivial ideal in $\mathbb{C}[[x, y]]$ and $\omega=x^{\nu-1} d x \wedge d y$ with $\nu \in \mathbb{N}^{*}$. If $\mathcal{I}$ is not divisible by $x$, we assume that $\nu=1$.

Consider the Newton polygon of $\mathcal{I}$ and its dual in $\mathbb{R}_{+}^{2}$. Here a face $S$ of the Newton polygon of $\mathcal{I}$ with equation $p \alpha+q \beta=N$ corresponds in the dual to a half-line going through the origin with direction $(p, q)$. This induces a decomposition of $\mathbb{R}_{+}^{2}$ into disjoint cones (of dimension 1 or 2 ).

(1) We denote by $\Delta$ such a 2-dimensional cone, and let it be generated by $\left(p_{1}, q_{1}\right)$ and $\left(p_{2}, q_{2}\right)$. Then we put

$$
\mathcal{P}_{\Delta}=\left\{(i, j) \in \mathbb{N}^{2} \mid i=\mu_{1} p_{1}+\mu_{2} p_{2}, j=\mu_{1} q_{1}+\mu_{2} q_{2},\left(\mu_{1}, \mu_{2}\right) \in \mathbb{Q}^{2}, 0<\mu_{1} \leq 1,0<\mu_{2} \leq 1\right\} .
$$

(2) We denote by $L$ such a 1-dimensional cone, and let it be generated by $(p, q)$.

Note that in (1) the set of points with integer coordinates in the cone $\Delta$ is precisely

$$
\left\{\left(k_{1}, k_{2}\right) \in \mathbb{N}^{2} \mid k_{1}=i_{0}+n_{1} p_{1}+n_{2} p_{2}, k_{2}=j_{0}+n_{1} q_{1}+n_{2} q_{2},\left(i_{0}, j_{0}\right) \in \mathcal{P}_{\Delta},\left(n_{1}, n_{2}\right) \in \mathbb{N}^{2}\right\} .
$$

In this context we recall the formula for the generating function of $\Delta$ :

$$
\sum_{\left(k_{1}, k_{2}\right) \in \Delta \cap \mathbb{N}^{2}} x_{1}^{k_{1}} x_{2}^{k_{2}}=\frac{\sum_{(i, j) \in \mathcal{P}_{\Delta}} x_{1}^{i} x_{2}^{j}}{\left(1-x_{1}^{p_{1}} x_{2}^{q_{1}}\right)\left(1-x_{1}^{p_{2}} x_{2}^{q_{2}}\right)} .
$$

And we put for later use

$$
D_{\Delta}=\sum_{(i, j) \in \mathcal{P}_{\Delta}} \mathbb{L}^{-(\nu i+j)} T^{i a+j b},
$$

where we denote by $(a, b)$ be the intersection point of the two faces of the Newton polygon whose supporting lines have equations $p_{1} \alpha+q_{1} \beta=N_{1}$ and $p_{2} \alpha+q_{2} \beta=N_{2}$.

We start the study of the contributions of elements $\left(k_{1}, k_{2}\right) \in \mathbb{N}^{2}$, depending on which cone they belong to.

(1) Assume that $\left(k_{1}, k_{2}\right)$ lies in a cone $\Delta$ generated by $\left(p_{1}, q_{1}\right),\left(p_{2}, q_{2}\right)$. Let $\phi \in \mathcal{L}^{0}\left(\mathbb{C}^{2}\right)$ :

$$
\phi(t)=\left\{\begin{array}{l}
x=c_{1} t^{k_{1}}+\cdots+a_{n} t^{n}+\cdots \\
y=c_{2} t^{k_{2}}+\cdots+b_{n} t^{n}+\cdots
\end{array}\right.
$$

where $c_{1}, c_{2} \in \mathbb{C}^{*}$, such that $\operatorname{ord}_{\phi} \mathcal{I}=n$. We have

$$
\begin{aligned}
& \operatorname{ord}_{\phi} \mathcal{I}=k_{1} a+k_{2} b=n, \\
& \operatorname{ord}(\omega \circ \phi)=(\nu-1) k_{1} .
\end{aligned}
$$

The contribution of $\Delta$ to $\zeta(\mathcal{I}, \omega)(T)$ is 


$$
\begin{aligned}
& (\mathbb{L}-1)^{2} \sum_{\left(k_{1}, k_{2}\right) \in \Delta \cap \mathbb{N}^{2}} \mathbb{L}^{n-k_{1}} \mathbb{L}^{n-k_{2}} \mathbb{L}^{-2 n} \mathbb{L}^{-(\nu-1) k_{1}} T^{n} \\
= & (\mathbb{L}-1)^{2} \sum_{\left(k_{1}, k_{2}\right) \in \Delta \cap \mathbb{N}^{2}} \mathbb{L}^{-\nu k_{1}-k_{2}} T^{k_{1} a+k_{2} b} \\
= & (\mathbb{L}-1)^{2} D_{\Delta} \sum_{\left(n_{1}, n_{2}\right) \in \mathbb{N}^{2}} \mathbb{L}^{-\left[\left(p_{1} \nu+q_{1}\right) n_{1}+\left(p_{2} \nu+q_{2}\right) n_{2}\right]} T^{\left(p_{1} a+q_{1} b\right) n_{1}+\left(p_{2} a+q_{2} b\right) n_{2}} \\
= & (\mathbb{L}-1)^{2} D_{\Delta} \frac{1}{\left(1-\mathbb{L}^{-\left(p_{1} \nu+q_{1}\right)} T^{N_{1}}\right)\left(1-\mathbb{L}^{-\left(p_{2} \nu+q_{2}\right)} T^{\left.N_{2}\right)}\right.} .
\end{aligned}
$$

(2) Assume that $\left(k_{1}, k_{2}\right)$ lies on a line $L$, generated by $(p, q)$. Then there exists $k \in \mathbb{N}^{*}$ such that $k_{1}=p k, k_{2}=q k$. Let $S$ be the corresponding face of the Newton polygon, with equation $p \alpha+q \beta=N$, and let $F_{S}$ be its face polynomial. Let $\phi \in \mathcal{L}^{0}\left(\mathbb{C}^{2}\right)$ :

$$
\phi(t)=\left\{\begin{array}{l}
x=c_{1} t^{p k}+\cdots+a_{n} t^{n}+\cdots \\
y=c_{2} t^{q k}+\cdots+b_{n} t^{n}+\cdots
\end{array}\right.
$$

where $c_{1}, c_{2} \in \mathbb{C}^{*}$, such that $\operatorname{ord}_{\phi} \mathcal{I}=n$. We have two cases.

(a) For any root $\mu$ of $F_{S}$, we have $c_{2}^{p}-\mu c_{1}^{q} \neq 0$. In this case,

$$
\begin{gathered}
\operatorname{ord}_{\phi} \mathcal{I}=N k, \\
\operatorname{ord}(\omega \circ \phi)=p(\nu-1) k .
\end{gathered}
$$

Denoting by $r$ the number of distinct roots of $F_{S}$, we compute the contribution of $L$ to $\zeta(\mathcal{I}, \omega)(T)$. It is equal to

$$
\left((\mathbb{L}-1)^{2}-C\right) \sum_{k \in \mathbb{N}^{*}} \mathbb{L}^{n-p k+n-q k-p(\nu-1) k} \mathbb{L}^{-2 n} T^{N k},
$$

where $C$ is the class in the Grothendieck ring of

$$
\left\{\left(c_{1}, c_{2}\right) \in\left(\mathbb{C}^{*}\right)^{2} \mid c_{2}^{p}-\mu_{j} c_{1}^{q}=0 \text { for some root } \mu_{j} \text { of } F_{S}\right\} .
$$

Since $\operatorname{gcd}(p, q)=1$, we have for each of the $r$ roots $\mu_{j}$ that the curve given by $Y^{p}-\mu_{j} X^{q}=0$ is parameterized by $u \mapsto\left(\mu_{j}^{q^{\prime}} u^{p}, \mu_{j}^{p^{\prime}} u^{q}\right)$, and hence that $C=$ $r(\mathbb{L}-1)$. We conclude that the contribution of $L$ is

$$
\begin{aligned}
& \left((\mathbb{L}-1)^{2}-r(\mathbb{L}-1)\right) \sum_{k \in \mathbb{N}^{*}} \mathbb{L}^{-(p \nu+q) k} T^{N k} \\
= & (\mathbb{L}-1)(\mathbb{L}-r-1) \frac{\mathbb{L}^{-(p \nu+q)} T^{N}}{1-\mathbb{L}^{-(p \nu+q)} T^{N}} .
\end{aligned}
$$

(b) There exists a root $\mu$ of $F_{l_{S}}$ such that $c_{2}^{p}-\mu c_{1}^{q}=0$. Fix such a root $\mu$. We need to compute

$$
\sum_{n \geq 1} \sum_{k \geq 1}\left[X_{n,(\nu-1) p k}\right] \mathbb{L}^{-2 n} \mathbb{L}^{-(\nu-1) p k} T^{n},
$$

where $X_{n,(\nu-1) p k}$ is the variety of $n$-jets $\phi$ :

$$
\phi(t)=\left\{\begin{array}{l}
x=a_{p k} t^{p k}+\cdots+a_{n} t^{n} \\
y=b_{q k} t^{t k}+\cdots+b_{n} t^{n}
\end{array}\right.
$$

such that $\operatorname{ord}_{\phi} \mathcal{I}=n, \operatorname{ord}(\omega \circ \phi)=(\nu-1) p k$ and $b_{q k}^{p}-\mu a_{p k}^{q}=0$. 
Denote by $\bar{X}_{n,(\nu-1) p k}$ the variety of $n$-jets $\bar{\phi}$ :

$$
\bar{\phi}(t)=\left\{\begin{array}{l}
\bar{\phi}_{1}=a_{0}^{\prime}+a_{1}^{\prime} t+\cdots+a_{n}^{\prime} t^{n} \\
\bar{\phi}_{2}=b_{0}^{\prime}+b_{1}^{\prime} t+\cdots+b_{n}^{\prime} t^{n}
\end{array}\right.
$$

such that $\left.\operatorname{ord}_{\left(t^{p k} \bar{\phi}_{1}, t q k\right.} \bar{\phi}_{2}\right) \mathcal{I}=n, \operatorname{ord}\left(\omega \circ\left(t^{p k} \bar{\phi}_{1}, t^{q k} \bar{\phi}_{2}\right)\right)=(\nu-1) p k$ and $\left(b_{0}^{\prime}\right)^{p}-$ $\mu\left(a_{0}^{\prime}\right)^{q}=0$. We have

$$
\left[X_{n,(\nu-1) p k}\right]=\left[\bar{X}_{n,(\nu-1) p k}\right] \mathbb{L}^{-p k-q k} .
$$

Next we consider the appropriate varieties of jets related to the Newton map $\sigma=\sigma_{(p, q, \mu)}$. Let $\omega_{1}=x_{1}^{p \nu+q-1} d x_{1} \wedge d y_{1}$. Denote by $\bar{X}_{n,(p \nu+q-1) k}^{\sigma}$ the variety of $n$-jets $\bar{\psi}$ :

$$
\bar{\psi}(t)=\left\{\begin{array}{lll}
\bar{\psi}_{1}= & r_{0}+r_{1} t+\cdots+r_{n} t^{n} \\
\bar{\psi}_{2}= & s_{1} t+\cdots+s_{n} t^{n}
\end{array}\right.
$$

such that $r_{0} \neq 0, \operatorname{ord}_{\left(t^{k} \bar{\psi}_{1}, \bar{\psi}_{2}\right)} \mathcal{I}_{\sigma}=n$ and $\operatorname{ord}\left(\omega_{1} \circ\left(t^{k} \bar{\psi}_{1}, \bar{\psi}_{2}\right)\right)=(p \nu+q-1) k$. Finally we denote by $X_{n,(p \nu+q-1) k}^{\sigma}$ the variety of $n$-jets $\psi$ :

$$
\left\{\begin{array}{l}
\psi_{1}=r_{k}^{\prime} t^{k}+\cdots+r_{n}^{\prime} t^{n} \\
\psi_{2}=s_{1}^{\prime} t+\cdots+s_{n}^{\prime} t^{n}
\end{array}\right.
$$

such that $r_{k}^{\prime} \neq 0, \operatorname{ord}_{\left(\psi_{1}, \psi_{2}\right)} \mathcal{I}_{\sigma}=n$ and $\operatorname{ord}\left(\omega_{1} \circ\left(\psi_{1}, \psi_{2}\right)\right)=(p \nu+q-1) k$. We have

$$
\left[\bar{X}_{n,(p \nu+q-1) k}^{\sigma}\right]=\left[X_{n,(p \nu+q-1) k}^{\sigma}\right] \mathrm{L}^{k} .
$$

We construct a map $S$ from $\bar{X}_{n,(p \nu+q-1) k}^{\sigma}$ to $\bar{X}_{n,(\nu-1) p k}$ in order to compare $\left[\bar{X}_{n,(\nu-1) p k}\right]$ and $\left[\bar{X}_{n,(p \nu+q-1) k}^{\sigma}\right]$.

For $\bar{\psi} \in \bar{X}_{n,(p \nu+q-1) k}$ given by

$$
\bar{\psi}(t)=\left\{\begin{array}{lll}
\psi_{1} & = & r_{0}+r_{1} t+\cdots+r_{n} t^{n} \\
\psi_{2} & = & s_{1} t+\cdots+s_{n} t^{n}
\end{array}\right.
$$

we define

$$
S(\bar{\psi})(t)=\left\{\begin{array}{l}
\bar{\phi}_{1}=\mu^{q^{\prime}} \bar{\psi}_{1}^{p} \bmod t^{n+1} \\
\bar{\phi}_{2}=\bar{\psi}_{1}^{q}\left(\bar{\psi}_{2}+\mu^{p^{\prime}}\right) \bmod t^{n+1} .
\end{array}\right.
$$

Then one easily verifies that

$$
\operatorname{ord}_{\left(t^{k} \bar{\psi}_{1}, \bar{\psi}_{2}\right)} \mathcal{I}_{\sigma}=n=\operatorname{ord}_{\left(t^{p k} \bar{\phi}_{1}, t t^{k} \bar{\phi}_{2}\right)} \mathcal{I} \text {. }
$$

The constant term of $\bar{\phi}_{1}$ is $a=\mu^{q^{\prime}} r_{0}^{p}$ and the constant term of $\bar{\phi}_{2}$ is $b=\mu^{p^{\prime}} r_{0}^{q}$. Hence we have $b^{p}=\mu a^{q}$. Note also that $S$ is clearly a morphism.

Lemma 3.3. The map $S$ defines a isomorphism between $\bar{X}_{n,(p \nu+q-1) k}^{\sigma}$ and $\bar{X}_{n,(\nu-1) p k}$.

Proof. We construct an inverse map. Take

$$
\bar{\phi}(t)=\left\{\begin{array}{l}
\bar{\phi}_{1}(t)=a_{0}^{\prime}+a_{1}^{\prime} t+\cdots+a_{n}^{\prime} t^{n}=a_{0}^{\prime} \tilde{\phi}_{1}(t) \\
\bar{\phi}_{2}(t)=b_{0}^{\prime}+b_{1}^{\prime} t+\cdots+b_{n}^{\prime} t^{n}=b_{0}^{\prime} \tilde{\phi}_{2}(t)
\end{array}\right.
$$

in $\bar{X}_{n,(\nu-1) p k}$. Then

$$
\bar{\psi}(t)=\left\{\begin{array}{l}
\bar{\psi}_{1}=\quad a_{0}^{p^{\prime}} / b_{0}^{q^{\prime}}\left(\tilde{\phi}_{1}\right)^{1 / p} \bmod t^{n+1} \\
\bar{\psi}_{2}=-\mu^{p^{\prime}}+\mu^{p^{\prime}} \tilde{\phi}_{2} /\left(\tilde{\phi}_{1}\right)^{q / p} \bmod t^{n+1}
\end{array}\right.
$$


belongs to $\bar{X}_{n,(p \nu+q-1) k}^{\sigma}$. Here $\left(\tilde{\phi}_{1}\right)^{1 / p}$ is well-defined and given by the standard expansion since the constant term of $\tilde{\phi}_{1}$ is 1 . One easily verifies that this map $\bar{\phi} \mapsto \bar{\psi}$ is indeed the inverse of $S$.

With these preparations we can compute

$$
\begin{aligned}
& \sum_{n \geq 1} \sum_{k \geq 1}\left[X_{n,(\nu-1) p k}\right] \mathbb{L}^{-(\nu-1) p k} \mathbb{L}^{-2 n} T^{n} \\
= & \sum_{n \geq 1} \sum_{k \geq 1}\left[\bar{X}_{n,(\nu-1) p k}\right] \mathbb{L}^{-q k} \mathbb{L}^{-\nu p k} \mathbb{L}^{-2 n} T^{n} \\
= & \sum_{n \geq 1} \sum_{k \geq 1}\left[\bar{X}_{n,(p \nu+q-1) k}^{\sigma}\right] \mathbb{L}^{-(q+p \nu) k} \mathbb{L}^{-2 n} T^{n} \\
= & \sum_{n \geq 1} \sum_{k \geq 1}\left[X_{n,(p \nu+q-1) k}^{\sigma}\right] \mathbb{L}^{-(p \nu+q-1) k} \mathbb{L}^{-2 n} T^{n} \\
= & \sum_{n \geq 1}\left(\sum_{m} \mu\left(V_{n, m}^{\sigma}\right) \mathbb{L}^{-m}\right) T^{n},
\end{aligned}
$$

where $V_{n, m}^{\sigma}$ is the set of $\operatorname{arcs} \phi \in \mathcal{L}^{0}\left(\mathbb{C}^{2}\right)$ such that $\operatorname{ord}_{\phi} \mathcal{I}_{\sigma}=n$ and $\operatorname{ord}\left(\omega_{1} \circ \phi\right)=$ $m$. This last expression is precisely $\zeta\left(\mathcal{I}_{\sigma}, \omega_{1}\right)(T)$.

We have proven the following formula.

Theorem 3.4. Let $\mathcal{I}$ and $\omega$ be as in definition 3.2. With the notation introduced there, we have

$$
\begin{aligned}
\zeta(\mathcal{I}, \omega)(T)= & \sum_{\Delta}(\mathbb{L}-1)^{2} D_{\Delta} \frac{1}{\left(1-\mathbb{L}^{-\left(p_{1} \nu+q_{1}\right)} T^{N_{1}}\right)\left(1-\mathbb{L}^{-\left(p_{2} \nu+q_{2}\right)} T^{N_{2}}\right)} \\
& +\sum_{L}(\mathbb{L}-1)(\mathbb{L}-r-1) \frac{\mathbb{L}^{-(p \nu+q)} T^{N}}{1-\mathbb{L}^{-(p \nu+q)} T^{N}} \\
& +\sum_{L} \sum_{\mu} \zeta\left(\mathcal{I}_{\sigma_{(p, q, \mu)}}, \omega_{1}\right)(T),
\end{aligned}
$$

where $\mu$ ranges over the roots of the face polynomial of the face corresponding to the cone $L$, and $\omega_{1}=x_{1}^{p \nu+q-1} d x_{1} \wedge d y_{1}$.

Example 1 (continued). The first Newton polygon gives rise in the dual plane to a decomposition of $\mathbb{R}_{+}^{2}$ in three (2-dimensional) cones and two lines. The cones are $\Delta_{0}$ generated by $(1,0)$ and $(2,1), \Delta_{1}$ generated by $(2,1)$ and $(1,1)$, and $\Delta_{2}$ generated by $(1,1)$ and $(0,1)$. The lines are $L_{1}$ generated by $(2,1)$, and $L_{2}$ generated by $(1,1)$.

The contribution of the cones to the zeta function for $\mathcal{I}$ and $\omega=x^{\nu-1} d x \wedge d y$ is

$$
\begin{aligned}
(\mathbb{L}-1)^{2}\left(\frac{\mathbb{L}^{-(3 \nu+1)} T^{5}}{\left(1-\mathbb{L}^{-(2 \nu+1)} T^{5}\right)\left(1-\mathbb{L}^{-\nu}\right)}\right. & +\frac{\mathbb{L}^{-(3 \nu+2)} T^{9}}{\left(1-\mathbb{L}^{-(2 \nu+1)} T^{5}\right)\left(1-\mathbb{L}^{-(\nu+1)} T^{4}\right)} \\
& \left.+\frac{\mathbb{L}^{-(\nu+2)} T^{4}}{\left(1-\mathbb{L}^{-(\nu+1)} T^{4}\right)\left(1-\mathbb{L}^{-1}\right)}\right) .
\end{aligned}
$$

The contribution of the lines is

$$
(\mathbb{L}-1)(\mathbb{L}-2)\left(\frac{\mathbb{L}^{-(2 \nu+1)} T^{5}}{\left(1-\mathbb{L}^{-(2 \nu+1)} T^{5}\right)}+\frac{\mathbb{L}^{-(\nu+1)} T^{4}}{\left(1-\mathbb{L}^{-(\nu+1} T^{4}\right)}\right) .
$$

We have considered the Newton map $\sigma_{(2,1,3)}$ given by

$$
x=3 x_{1}^{2}, \quad y=x_{1}\left(y_{1}+3\right),
$$




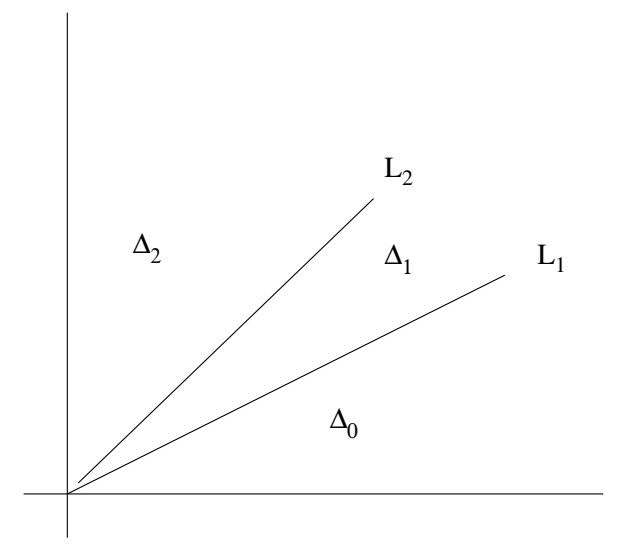

FIGURE 3.

with image ideal $\mathcal{I}_{1}=\left(x_{1}^{5} y_{1}\right)$. We have $\omega_{1}=x_{1}^{2 \nu} d x_{1} \wedge d y_{1}$.

The contribution of the ideal $\mathcal{I}_{1}$ is

$$
(\mathbb{L}-1)^{2} \frac{\mathbb{L}^{-(2 \nu+1)} T^{5}}{\left(1-\mathbb{L}^{-(2 \nu+1)} T^{5}\right)} \frac{\mathbb{L}^{-1} T}{\left(1-\mathbb{L}^{-1} T\right)} .
$$

Next we consider the Newton map $\sigma_{(p, q, \mu)}=\sigma_{(1,1,-1)}$ associated to $S_{2}$ and $\mu=-1$. It is given by the substitution

$$
x=x_{1}, \quad y=x_{1}\left(y_{1}-1\right),
$$

with image ideal given by

$$
\mathcal{I}_{1}=x_{1}^{4}\left(x_{1}^{2} y_{1}, y_{1}^{3}+x_{1}^{5}\right)
$$

Its Newton polygon is given in Figure 2. We have $\omega_{1}=x_{1}^{\nu} d x_{1} \wedge d y_{1}$.

We have again three (2-dimensional) cones in the dual space: $\Delta_{0}^{\prime}$ generated by $(1,0)$ and $(1,1), \Delta_{1}^{\prime}$ generated by $(1,1)$ and $(1,3)$, and $\Delta_{2}^{\prime}$ generated by $(1,3)$ and $(0,1)$. The contribution of theses cones to the motivic zeta function is

$$
\begin{aligned}
(\mathbb{L}-1)^{2}\left(\frac{\mathbb{L}^{-(2 \nu+3)} T^{11}}{\left(1-\mathbb{L}^{-(\nu+2)} T^{7}\right)\left(1-\mathbb{L}^{-(\nu+1)} T^{4}\right)}\right. & +\frac{\left(\mathbb{L}^{-(\nu+3)} T^{8}+\mathbb{L}^{-(2 \nu+6)} T^{16}\right.}{\left(1-\mathbb{L}^{-(\nu+2)} T^{7}\right)\left(1-\mathbb{L}^{-(\nu+4)} T^{9}\right)} \\
& \left.+\frac{\mathbb{L}^{-(\nu+5)} T^{9}}{\left(1-\mathbb{L}^{-(\nu+4)} T^{9}\right)\left(1-\mathbb{L}^{-1}\right)}\right) .
\end{aligned}
$$

The two faces are dicritical faces. The contribution of the two lines is

$$
(\mathbb{L}-1)^{2}\left(\frac{\mathbb{L}^{-(\nu+2)} T^{7}}{\left(1-\mathbb{L}^{-(\nu+2)} T^{7}\right)}+\frac{\mathbb{L}^{-(\nu+4)} T^{9}}{\left(1-\mathbb{L}^{-(\nu+4)} T^{9}\right)}\right) .
$$

The final calculation gives

$$
\zeta(\mathcal{I}, \omega)(T)=\frac{P\left(\mathbb{L}^{-1}, T\right)}{\left.\left(1-\mathbb{L}^{-(\nu+4)} T^{9}\right)\right)\left(1-\mathbb{L}^{-(\nu+2)} T^{7}\right)\left(1-\mathbb{L}^{-1} T\right)},
$$

where $P\left(\mathbb{L}^{-1}, T\right)$ is a polynomial.

We observe that all faces of the successive Newton polygons contribute to the computation of the motivic zeta function. A face with equation $p \alpha+q \beta=N$ gives rise to a factor in the denominators of the contributions of the form $\left(1-\mathbb{L}^{-(p \nu+q)} T^{N}\right)$, where the differential form at that stage of the Newton algorithm has the form $\omega=x^{\nu-1} d x \wedge d y$. However, at the end of the computation not all of these factors remain. We prove a general result concerning this phenomenon. 
Proposition 3.5. Take $\mathcal{I}$ and $\omega$ as in Theorem 3.4. Let $S$ be a face of the Newton polygon of $\mathcal{I}$ with equation $\alpha+q \beta=N$ that hits the $x$-axis, which is not a dicritical face and such that its face polynomial has exactly one root. Then $\left(1-\mathbb{L}^{-(\nu+q)} T^{N}\right)$ does not appear in the denominator of the motivic zeta function of $\mathcal{I}$ and $\omega$. The analogous result holds for a face $p \alpha+\beta=N$ that hits the $y$-axis, with the same hypothesis.
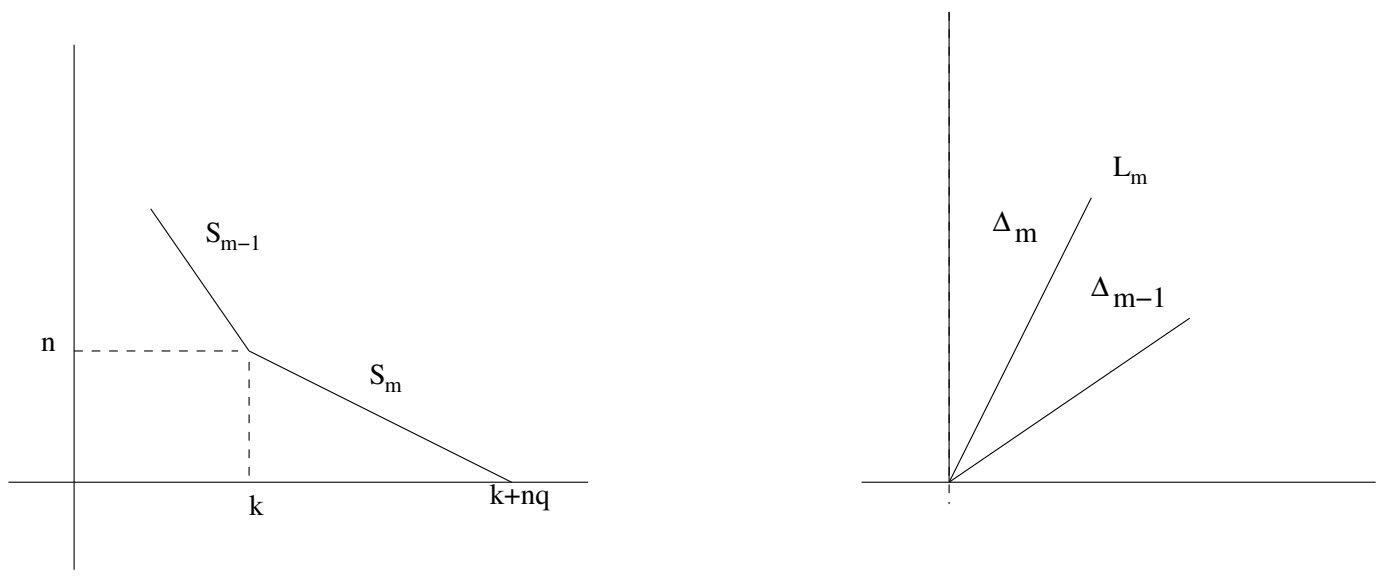

\section{FIGURE 4.}

Proof. We assume the face $S=S_{m}$ has equation $\alpha+q \beta=N$. Its extremities have coordinates of the form $(k, n)$ and $(k+n q, 0)$ with $N=k+n q$. Writing the equation of the face $S_{m-1}$ as $p_{m-1} \alpha+q_{m-1} \beta=N_{m-1}$, we have $N_{m-1}=p_{m-1} k+q_{m-1} n$. If $k=0$, the face $S_{m-1}$ has equation $\alpha=0$.

There are four contributions of the face $S_{m}$ to the zeta function. The contribution of $\Delta_{m}$ is

$$
(\mathbb{L}-1)^{2} \frac{\mathbb{L}^{-(1+\nu+q)} T^{N}}{\left(1-\mathbb{L}^{-1}\right)\left(1-\mathbb{L}^{-(\nu+q)} T^{N}\right)}
$$

(noting that $\mathcal{P}_{\Delta_{m}}=\{(1, q+1)\}$ ), and the contribution of $L_{m}$ is

$$
(\mathbb{L}-1)(\mathbb{L}-2) \frac{\mathbb{L}^{-(\nu+q)} T^{N}}{\left(1-\mathbb{L}^{-(\nu+q)} T^{N}\right)} .
$$

The sum of these two contributions is

where

$$
\begin{aligned}
\zeta_{1}(\mathcal{I}, \omega)(T) & =(\mathbb{L}-1)^{2} \frac{\mathbb{L}^{-(\nu+q)} T^{N}}{\left(1-\mathbb{L}^{-(\nu+q)} T^{N}\right)} \\
& =\left.(\mathbb{L}-1)^{2} \Phi_{1}\left(x_{1}, x_{2}\right)\right|_{x_{1}=\mathbb{L}^{-\nu} T^{k}, x_{2}=\mathbb{L}^{-1} T^{n}}
\end{aligned}
$$

The contribution of the cone $\Delta_{m-1}$ is

$$
\Phi_{1}\left(x_{1}, x_{2}\right)=\frac{x_{1} x_{2}^{q}}{1-x_{1} x_{2}^{q}} .
$$

$$
\begin{aligned}
\zeta_{2}(\mathcal{I}, \omega)(T) & =(\mathbb{L}-1)^{2} \frac{D_{\Delta_{m-1}}}{\left(1-\mathbb{L}^{-(\nu+q)} T^{N}\right)\left(1-\mathbb{L}^{-\left(p_{m-1} \nu+q_{m-1}\right)} T^{N_{m-1}}\right)} \\
& =\left.(\mathbb{L}-1)^{2} \Phi_{2}\left(x_{1}, x_{2}\right)\right|_{x_{1}=\mathbb{L}^{-\nu} T^{k}, x_{2}=\mathbb{L}^{-1} T^{n}},
\end{aligned}
$$

where

$$
\Phi_{2}\left(x_{1}, x_{2}\right)=\frac{D\left(x_{1}, x_{2}\right)}{\left(1-x_{1} x_{2}^{q}\right)\left(1-x_{1}^{p_{m-1}} x_{2}^{q_{m-1}}\right)}
$$


and

$$
D\left(x_{1}, x_{2}\right)=\sum_{(i, j) \in \mathcal{P}_{\Delta_{m-1}}} x_{1}^{i} x_{2}^{j} .
$$

The remaining part arises after applying the Newton map $\sigma_{(1, q, \mu)}$ :

$$
x=x_{1}, \quad y=x_{1}^{q}\left(y_{1}+\mu\right),
$$

where $\mu$ is the root of $F_{S_{m}}$. We have $\omega_{1}=x_{1}^{\nu+q-1} d x_{1} \wedge d y_{1}$.
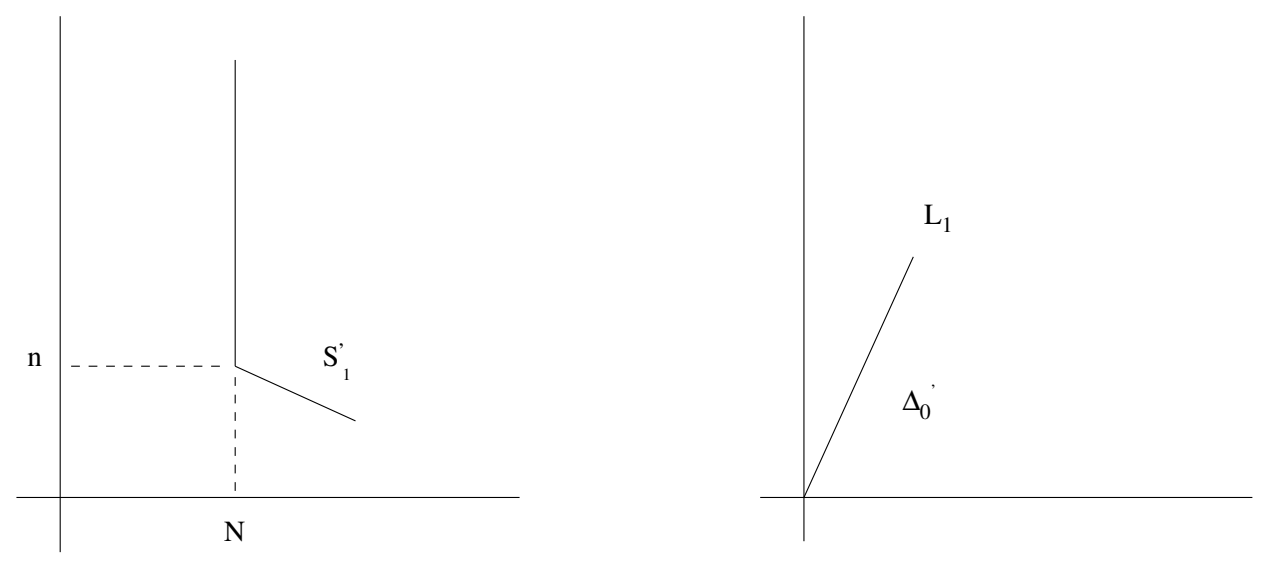

FiguRE 5.

The contribution to the zeta function comes from the cone $\Delta_{0}^{\prime}$ for the new Newton polygon. Writing the equation of the new face $S_{1}^{\prime}$ as $p_{1}^{\prime} \alpha+q_{1}^{\prime} \beta=N_{1}^{\prime}$, we have $N_{1}^{\prime}=p_{1}^{\prime} N+q_{1}^{\prime} n$. Then this contribution is

$$
\zeta_{3}(\mathcal{I}, \omega)(T)=(\mathbb{L}-1)^{2} \frac{D_{\Delta_{0}^{\prime}}}{\left(1-\mathbb{L}^{-(\nu+q)} T^{N}\right)\left(1-\mathbb{L}^{-\left(p_{1}^{\prime}(\nu+q)+q_{1}^{\prime}\right)} T^{\left.N_{1}^{\prime}\right)}\right.},
$$

where

$$
D_{\Delta_{0}^{\prime}}=\sum_{(i, j) \in \mathcal{P}_{\Delta_{0}^{\prime}}}\left(\mathbb{L}^{-(\nu+q)} T^{N}\right)^{i}\left(\mathbb{L}^{-1} T^{n}\right)^{j}
$$

We can write

$$
\left(\mathbb{L}^{-(\nu+q)} T^{N}\right)^{i}\left(\mathbb{L}^{-1} T^{n}\right)^{j}=\left(\mathbb{L}^{-\nu} T^{k}\right)^{i}\left(\mathbb{L}^{-1} T^{n}\right)^{j+q i}
$$

and similarly

$$
\begin{gathered}
1-\mathbb{L}^{-(\nu+q)} T^{N}=1-\mathbb{L}^{-\nu} T^{k}\left(\mathbb{L}^{-1} T^{n}\right)^{q}, \\
1-\mathbb{L}^{-\left((\nu+q) p_{1}^{\prime}+q_{1}^{\prime}\right)} T^{N_{1}^{\prime}}=1-\left(\mathbb{L}^{-\nu} T^{k}\right)^{p_{1}^{\prime}}\left(\mathbb{L}^{-1} T^{n}\right)^{q_{1}^{\prime}+p_{1}^{\prime} q} .
\end{gathered}
$$

Then

where

$$
\zeta_{3}(\mathcal{I}, \omega)(T)=\left.(\mathbb{L}-1)^{2} \Phi_{3}\left(x_{1}, x_{2}\right)\right|_{x_{1}=\mathbb{L}^{-\nu} T^{k}, x_{2}=\mathbb{L}^{-1} T^{n}},
$$

and

$$
\Phi_{3}\left(x_{1}, x_{2}\right)=\frac{D_{0}\left(x_{1}, x_{2}\right)}{\left(1-x_{1} x_{2}^{q}\right)\left(1-x_{1}^{p_{1}^{\prime}} x_{2}^{q_{1}^{\prime}+p_{1}^{\prime} q}\right)}
$$

$$
D_{0}\left(x_{1}, x_{2}\right)=\sum_{(i, j) \in \mathcal{P}_{\Delta_{0}^{\prime}}}\left(x_{1}\right)^{i}\left(x_{2}\right)^{j+i q} .
$$

The rational function $\Phi_{1}\left(x_{1}, x_{2}\right)$ is the generating function of the cone generated by $(1, q)$, the rational function $\Phi_{2}\left(x_{1}, x_{2}\right)$ is the generating function of the cone generated by $(1, q)$ and $\left(p_{m-1}, q_{m-1}\right)$, and the rational function $\Phi_{3}\left(x_{1}, x_{2}\right)$ is the generating function of the cone 
generated by $(1, q),\left(p_{1}^{\prime}, q_{1}^{\prime}+q p_{1}^{\prime}\right)$. The map $(i, j) \rightarrow\left(i^{\prime}=i, j^{\prime}=j+q i\right)$ being a linear automorphism of the plane, we see that the configuration is as in Figure 6.

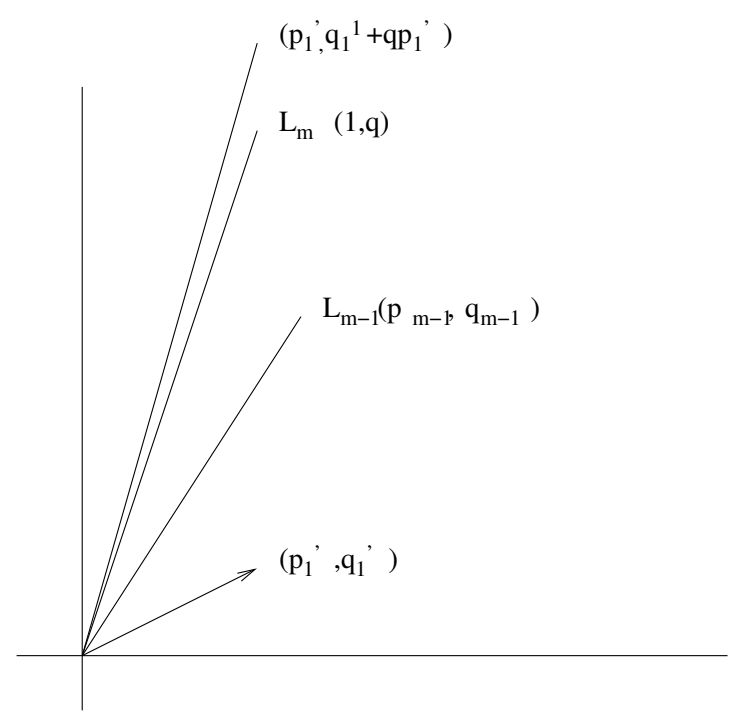

FiguRE 6.

Hence

$$
\Phi_{1}\left(x_{1}, x_{2}\right)+\Phi_{2}\left(x_{1}, x_{2}\right)+\Phi_{3}\left(x_{1}, x_{2}\right)=\Phi_{4}\left(x_{1}, x_{2}\right),
$$

where $\Phi_{4}\left(x_{1}, x_{2}\right)$ is the generating function of the cone $\Delta$ generated by $\left(p_{1}^{\prime}, q_{1}^{\prime}+q p_{1}^{\prime}\right)$ and $\left(p_{m-1}, q_{m-1}\right)$.

Then finally the sum of the four contributions is equal to

$$
\left.(\mathbb{L}-1)^{2} \Phi_{4}\left(x_{1}, x_{2}\right)\right|_{x_{1}=\mathbb{L}^{-\nu} T^{k}, x_{2}=\mathbb{L}^{-1} T^{n},}
$$

where

$$
\Phi_{4}\left(x_{1}, x_{2}\right)=\frac{\sum_{(i, j) \in \mathcal{P}_{\Delta}} x_{1}^{i} x_{2}^{j}}{\left(1-x_{1}^{p_{1}^{\prime}} x_{2}^{q_{1}^{\prime}+q p_{1}^{\prime}}\right)\left(1-x_{1}^{p_{m-1}} x_{2}^{q_{m}-1}\right)}
$$

Remark 3.6. We comment on two special cases above.

(1) When $S$ is the only face of the Newton polygon, the proof is just easier.

(2) It is possible that one of the two factors in the denominator of $\Phi_{4}$ is, after substitution, equal to $1-\mathbb{L}^{-(\nu+q)} T^{N}$. In the statement of the proposition we mean that the factor coming from $S$ cancels.

\section{NEWTON TREe ASSOciated With AN IDEAL}

In [7] we collected the information of the Newton algorithm of an ideal in its Newton tree. It reflects the 'tree shape' of the algorithm, and keeps the information on the successive Newton polygons. We recall briefly the main ideas. 
4.1. Graph associated with a Newton diagram. A graph associated with a Newton diagram is a vertical linear graph with vertices, edges connecting vertices and two arrows at the top and the bottom.

If the Newton polygon is empty, that is, $\Delta=(N, M)+\mathbb{R}_{+}^{2}$, the graph is in Figure 7 . It has one edge connecting two arrows decorated by $N$ and $M$ at the top and the bottom, respectively.

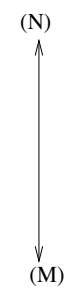

\section{FIGURE 7.}

If the Newton polygon is $\cup_{1 \leq i \leq m} S_{i}$, the graph has $m$ vertices $v_{1}, \cdots, v_{m}$ representing the faces $S_{1}, \cdots, S_{m}$. They are connected by edges when the faces intersect. We add one edge at $v_{1}$ and at $v_{m}$ ended by an arrow.

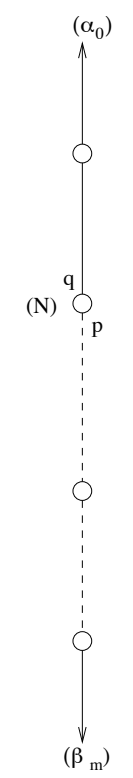

\section{FiguRE 8.}

We decorate the vertices and the extremities of the edges near the vertices using the following rule. Let $v$ be a vertex and $S$ be the corresponding face whose supporting line has equation $p \alpha+q \beta=N$, where $(p, q) \in\left(\mathbb{N}^{*}\right)^{2}$ and $\operatorname{gcd}(p, q)=1$. We decorate the vertex by $(N)$. Further we decorate the extremity of the edge above the vertex with $q$ and the extremity of the edge under the vertex by $p$; we say that the decorations near $v$ are $(q, p)$. The arrows represent the non-compact faces with supporting lines $\left\{x=\alpha_{0}\right\}$ and $\left\{y=\beta_{m}\right\}$; they are decorated with $\left(\alpha_{0}\right)$ at the top and $\left(\beta_{m}\right)$ at the bottom. See Figure 8.

4.2. Newton tree of an ideal. We build the Newton tree of $\mathcal{I}$ by induction on the depth. If its depth is zero, the ideal is generated by a 'monomial' $x^{N}(y+h(x))^{M}$; we define its Newton tree to be the graph as in Figure 7 . Let now $\mathcal{I}$ be an ideal of depth $d(\mathcal{I})$ greater 
than or equal to one. We assume that we are able to construct the Newton trees of ideals of depths $d<d(\mathcal{I})$.

On one hand we have the graph of the Newton polygon of the ideal $\mathcal{I}$. Consider a vertex $v$ on this graph. It is associated with a face $S$ of the Newton polygon of $\mathcal{I}$ with equation $p_{S} \alpha+q_{S} \beta=N_{S}$ and

$$
\operatorname{in}(\mathcal{I}, S)=x^{a_{S}} y^{b_{S}} F_{\mathcal{I}, S}\left(x^{q_{S}}, y^{p_{S}}\right)\left(k_{1}\left(x^{q_{S}}, y^{p_{S}}\right), \cdots, k_{s}\left(x^{q_{S}}, y^{p_{S}}\right)\right)
$$

with $\operatorname{deg} k_{i}=d_{S} \geq 0$. We decorate the vertex $v$ with the pair $\left(N_{S}, d_{S}\right) \in \mathbb{N}^{2}$.

Now we apply the Newton maps $\sigma=\sigma_{\left(p_{S}, q_{S}, \mu_{i}\right)}$ for each root $\mu_{i}$ of the face polynomial. (If the face is dicritical we already know that the maps $\sigma_{\left(p_{S}, q_{S}, \mu\right)}$ for $\mu$ generic give a monomial ideal of the form $\left(x^{N_{S}}\right)$ and we don't need to perform those Newton maps.) The transformed ideal $\mathcal{I}_{\sigma}$ has depth less than $d(\mathcal{I})$. Then from the induction hypothesis we can construct the Newton tree of $\mathcal{I}_{\sigma}$. It has a top arrow decorated with $N_{S}$. We delete this arrow and glue the edge on the vertex $v$. The edge which is glued on the vertex $v$ is a horizontal edge. Horizontal edges join vertices corresponding to different Newton polygons and vertical edges join vertices corresponding to the same Newton polygon. Note that the 'width' of the Newton tree of $\mathcal{I}$ is precisely its depth $d(\mathcal{I})$.

We explain now how we decorate the Newton tree. Let $v$ be a vertex on the Newton tree of $\mathcal{I}$. If $v$ corresponds to a face of the Newton polygon of $\mathcal{I}$, we say that $v$ has no preceding vertex and we define $\mathcal{S}(v)=\{v\}$. If $v$ does not correspond to a face of the Newton polygon of $\mathcal{I}$, it corresponds to a face of the Newton polygon of some $\mathcal{I}_{\Sigma}$. The Newton tree of $\mathcal{I}_{\Sigma}$ has been glued on a vertex $v_{1}$ which is called the preceding vertex of $v$. We note that the path between one vertex and its preceding vertex contains exactly one horizontal edge but may contain some vertical edges, for example as in Figure 9.

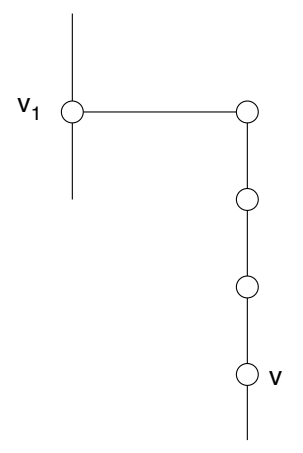

FiguRE 9.

If $v_{1}$ does not correspond to a face of the polygon of $\mathcal{I}$, we can consider its preceding vertex, and so on. We define $\mathcal{S}(v)=\left\{v_{i}, \cdots, v_{2}, v_{1}, v\right\}$ where $v_{j}, 2 \leq j \leq i$, is the preceding vertex of $v_{j-1}$, and $v_{i}$ corresponds to a face of the Newton polygon of $\mathcal{I}$. The final Newton tree is decorated in the following way. Let $v$ be a vertex on the Newton tree of $\mathcal{I}$. If $\mathcal{S}(v)=\{v\}$, the decorations near $v$ are not changed. If $\mathcal{S}(v)=\left\{v_{i}, \cdots, v_{2}, v_{1}, v\right\}$ and if the edge decorations near $v$ on the Newton tree where $\mathcal{S}(v)=\left\{v_{i-1}, \cdots, v_{2}, v_{1}, v\right\}$ are $(m, p)$, then after the gluing on $v_{i}$ they become $\left(m+p_{i} q_{i} p_{i-1}^{2} \cdots p_{1}^{2} p, p\right)$. The decorations of the vertices and of the arrows are not changed. Usually we do not write the decoration of arrows decorated with (1). We refer to [7] for motivations and applications.

The vertices decorated with $(N, d)$ with $d>0$ (corresponding to dicritical faces) are called dicritical vertices.

In $§ 5$ we will use the notion of edge determinant of an edge $e$; this is the product of the edge decorations on $e$ minus the product of the adjacent edge decorations to $e$. 
Example 1 (continued). In Figure 10 we draw the graphs associated with the occurring Newton diagrams, and the resulting Newton tree.

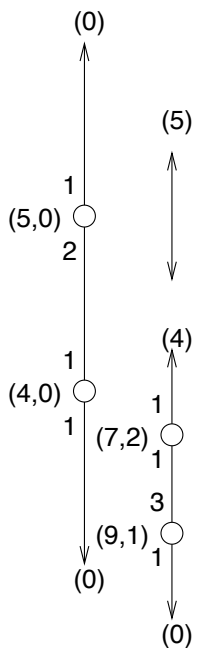

FIGURE 10.

4.3. Minimal Newton trees. The construction of the Newton trees shows that each vertex of the Newton tree of an ideal $\mathcal{I}$ corresponds to a face of a Newton polygon appearing in the Newton algorithm.

If the decorations near a vertex are $(q, p)$ and the decoration of the vertex is $N$, then there is a possible contribution of the vertex to the denominator of the motivic zeta function, namely $\left(1-\mathbb{L}^{-(\nu p+q)} T^{N}\right)$. However, Proposition 3.5 shows that certain vertices do not contribute. Those vertices correspond to faces of the Newton polygon which are not dicritical faces, have equation $\alpha+q \beta=N$ or $p \alpha+\beta=N$, hit respectively the $x$-axis or the $y$-axis, and have a face polynomial with only one root. Such a vertex is the top vertex (on the first vertical line) if its nearby decorations are $(1, p)$, it is not dicritical and has valency 3 , and the top arrow is decorated with (0). It is a vertex at the bottom of the tree if its nearby decorations are $(q, 1)$, it is not dicritical and has valency 3 , and the bottom arrow is decorated with (0). See Figure 11. Example 1 is an example with such vertices.

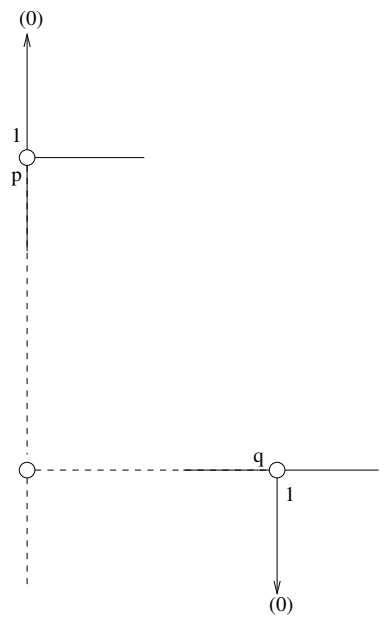

Figure 11. 
We will construct Newton trees where such vertices do not appear. For that purpose we first introduce certain 'manipulations' on trees. These will turn out to correspond to coordinate changes. The discussion will lead to a notion of minimal Newton tree, with a geometric interpretation developed in $§ 5$.

Take a vertex $v$ as above. Assume first that its nearby decorations are $(q, 1)$ such that either $q \neq 1$, or $q=1$ and $v$ is not connected to two arrows decorated with (0). The edge $e$ decorated with $q$ is connected to a vertex or an arrow, denoted by $v_{1}$. The edge $e_{v}$ decorated with 1 is connected to an arrow decorated with (0) and the horizontal edge $e_{h}$ is connected to a vertex or an arrow denoted by $v_{2}$. We delete the vertex $v$ and the edge $e_{v}$ with the arrow at the other end. We connect $v_{1}$ and $v_{2}$ by an edge with the same orientation as $e$ and we decorate with the decoration on $e$ near $v_{1}$ and with the decoration on $e_{h}$ near $v_{2}$. We call this operation erasing the vertex $v$. See Figure 12.
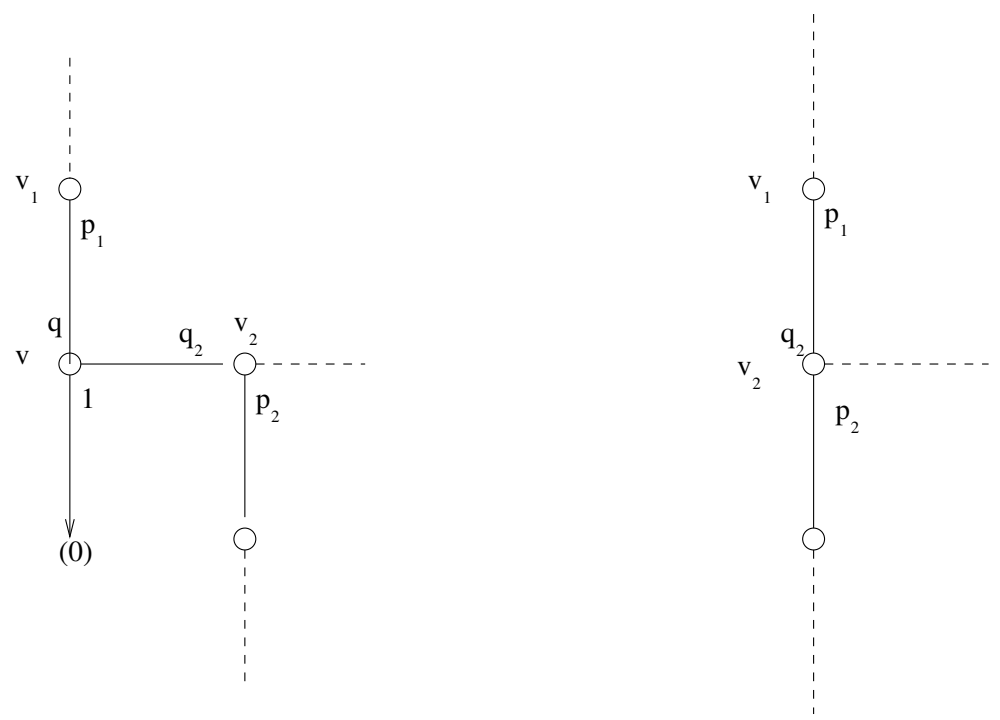

FiguRE 12.

We act symmetrically for the first top vertex if it is decorated with $(1, p)$ such that either $p \neq 1$, or $p=1$ and $v$ is not connected to two arrows decorated with (0). Note that in this case we change some decorations $\left(q_{2}, p_{2}\right)$ in $\left(p_{2}, q_{2}\right)$. See Figure 13.

Let finally $v$ be decorated with $(1,1)$ and connected to two arrows decorated with $(0)$. The vertex $v$ is connected to a vertex $v_{2}$ decorated with $\left(q_{2}, p_{2}\right)$ with $q_{2}>1$. We erase the vertex $v$ as well as the edge with the bottom arrow. The top vertex of the new Newton tree is $v_{2}$ decorated with $\left(q_{2}, p_{2}\right)$. See Figure 14 .

If above the vertex $v_{2}$ is a bottom vertex, with bottom arrow decorated by $(0)$, if it has valence 3 and is not dicritical, and if $p_{2}=1$, then we are in the previous case and we can also erase $v_{2}$. We can continue this way.

Definition 4.1. A Newton tree is minimal if the only vertices connected to an arrow decorated with (0) by an edge decorated with 1 are dicritical vertices.

In order to obtain a minimal Newton tree, starting from any Newton tree, erasing vertices (if applicable) is a good start. To finalize the procedure we introduce a more general operation on Newton trees that we call exchange of vertical edge.

Let $\mathcal{T}$ be a Newton tree. Assume we have a vertex $v$ decorated with $(q, 1)$. Let $e_{1}$ be the vertical edge arising from $v$ decorated with $p=1$ and let $v_{1}$ be the vertex or the arrow 

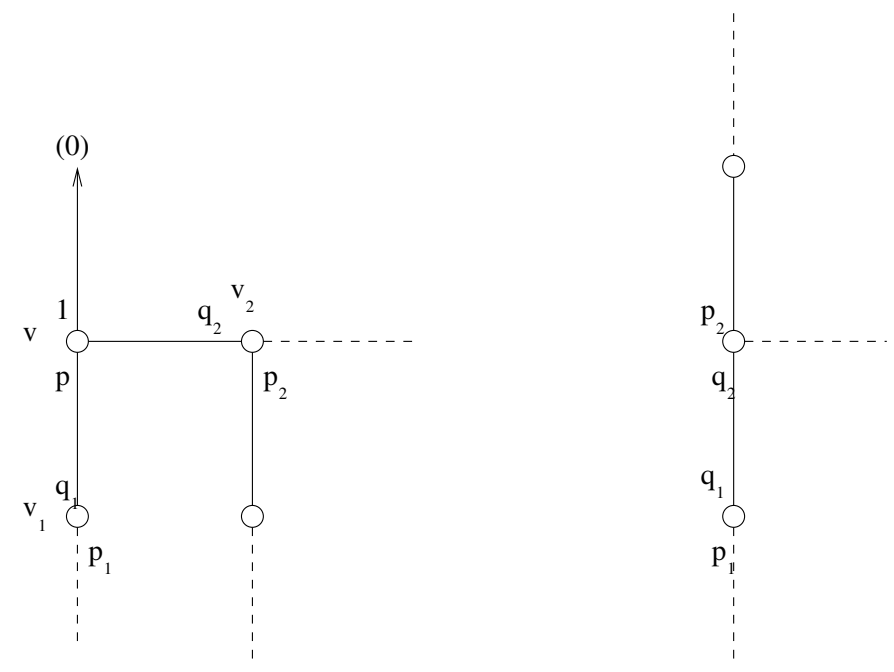

FiguRE 13.
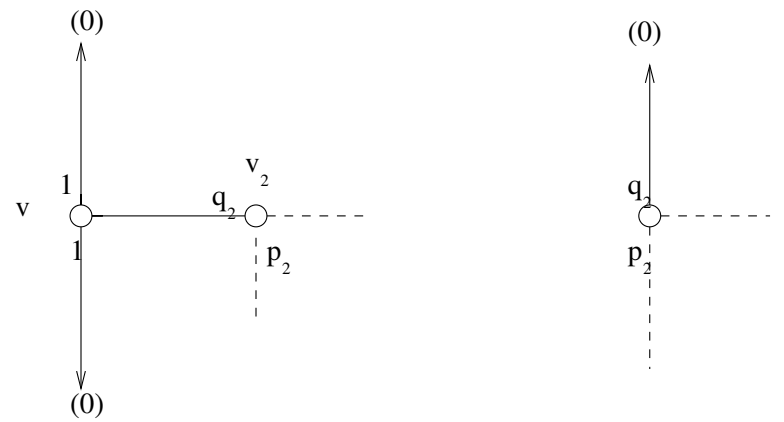

FIGURE 14.

at the other extremity of $e_{1}$. Choose a horizontal edge arising from $v$, denote it by $e_{2}$ and its other extremity by $v_{2}$. Let $\mathcal{T}^{\prime}$ be the tree obtained the following way. We cut $e_{1}$ and $e_{2}$ near the vertex $v$. Then we obtain three subtrees: the part $\mathcal{T}_{v}$ of $\mathcal{T}$ which contains $v$, the subtree $\mathcal{T}_{1}$ which contains $e_{1}$ and the subtree $\mathcal{T}_{2}$ which contains $e_{2}$. We stick $\mathcal{T}_{1}$ on $v$ by $e_{1}$ as a horizontal edge, not changing the rest of $\mathcal{T}_{1}$, and we stick $\mathcal{T}_{2}$ on $v$ by $e_{2}$ as a vertical edge, not changing the rest of $\mathcal{T}_{2}$. See Figure 15.
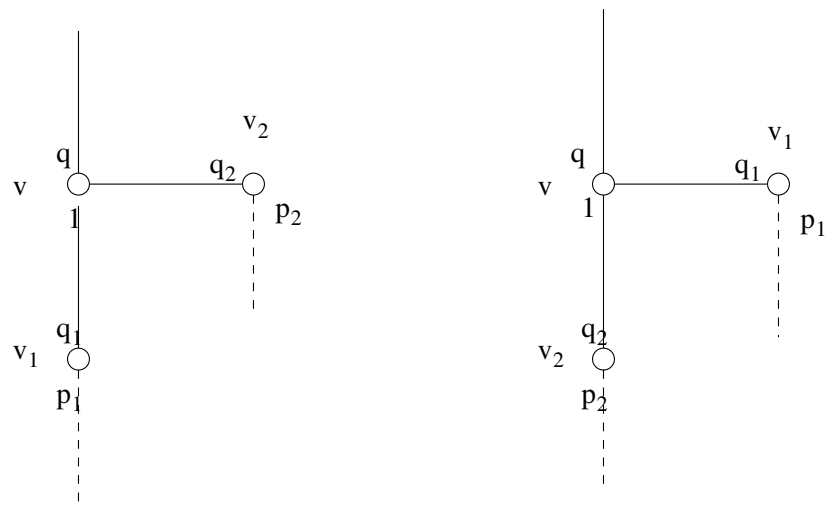

FIGURE 15. 
Remark 4.2. Erasing a vertex $v$ is a particular case of exchanging vertical edges. In fact, let $v$ be a vertex decorated with $(q, 1)$. We assume that $v$ is not a dicritical vertex. Let $e_{1}$ be the vertical edge decorated with 1 . We assume that $v_{1}$ is an arrow decorated with $(0)$. Assume there is only one horizontal edge $e_{2}$. Then we are in the case where we can erase $v$. We exchange the edges $e_{1}$ and $e_{2}$. We can erase now the horizontal edge because the arrow is decorated with (0) (In a Newton tree the horizontal edges are ending with vertices or with arrows decorated with strictly positive numbers.) Then we are left with a vertical edge with a vertex of valency 2 on it that we can erase. Exchange of vertical edge can also be used to erase a vertical edge decorated with 1 and ending with an arrow decorated with (0), see Figure 16.
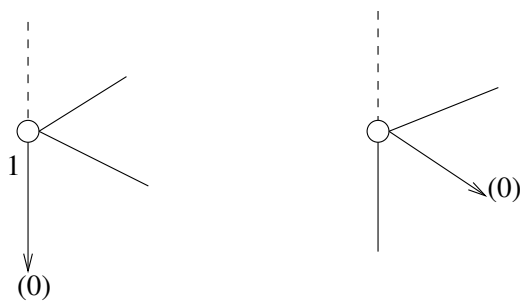

FiguRE 16.

As long as there exists a non-dicritical vertex that is connected to an arrow decorated with (0) by an edge decorated with 1 , we can clearly perform an exchange of vertical edge in order to erase it. Hence we showed the following.

Proposition 4.3. By exchanging vertical edges one can obtain a minimal Newton tree, starting from any Newton tree.

Example 1 (continued). A minimal Newton tree for the tree of Figure 10 is given in Figure 17.

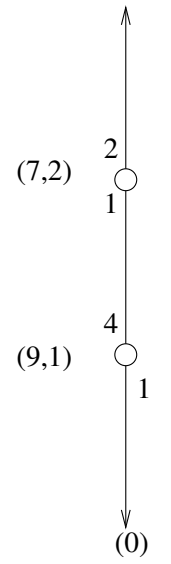

FIGURE 17.

Remark 4.4. A minimal Newton tree of the generic curve of an ideal may not coincide with a minimal Newton tree of the ideal. A dicritical vertex of degree 1 may be erased in the minimal Newton tree of the generic curve but is not to be erased in the minimal Newton tree of the ideal. 
Now we show that minimal Newton trees can be obtained as Newton trees in some coordinates.

Proposition 4.5. Let $\mathcal{I} \subset \mathbb{C}[[x, y]]$ be a non-trivial ideal with Newton tree $\mathcal{T}$. Assume there is on the first vertical line of $\mathcal{T}$ a vertex $v$ decorated with $(q, 1)$. Choose a horizontal edge to make an exchange of vertical edge. Let $\mathcal{T}^{\prime}$ be the resulting tree. Then there exists a change of coordinates $\phi:(x, y) \mapsto\left(x^{\prime}, y^{\prime}\right)$ such that $\mathcal{T}^{\prime}$ is the Newton tree of the ideal $\mathcal{I}^{\prime}=\phi(\mathcal{I}) \subset \mathbb{C}\left[\left[x^{\prime}, y^{\prime}\right]\right]$

A similar result holds for a vertex on the first vertical line decorated with $(1, p)$.

Proof. Let $S$ be the face of the Newton polygon corresponding to $v$. Write

$$
\operatorname{in}(\mathcal{I}, S)=x^{a_{S}} y^{b_{S}} F_{\mathcal{I}, S}\left(x^{q_{S}}, y\right)\left(k_{1}\left(x^{q_{S}}, y\right), \cdots, k_{s}\left(x^{q_{S}}, y\right)\right)
$$

with

$$
F_{\mathcal{I}, S}\left(x^{q_{S}}, y\right)=\prod_{1 \leq i \leq n}\left(y-\mu_{i} x^{q_{S}}\right)^{\nu_{i}}
$$

as in (2.1) or (2.2).

Consider first the case where the horizontal edge we have chosen does not end with an arrow. Let $\mu$ be the root (with multiplicity $\nu$ ) corresponding to the edge we have chosen. We perform the change of variables

$$
x^{\prime}=x, \quad y^{\prime}=y-\mu x^{q_{S}} .
$$

The faces above $S$ do not change. The face $S$ transforms into a face $S^{\prime}$ with the same $\left(q_{S}, 1\right)$. Its associated initial ideal is

$$
\operatorname{in}\left(\mathcal{I}, S^{\prime}\right)=\left(x^{\prime}\right)^{a_{S}}\left(y^{\prime}\right)^{\nu} F_{\mathcal{I}, S^{\prime}}\left(\left(x^{\prime}\right)^{q_{S}}, y^{\prime}\right)\left(k_{1}^{\prime}\left(\left(x^{\prime}\right)^{q_{S}}, y^{\prime}\right), \cdots, k_{s}^{\prime}\left(\left(x^{\prime}\right)^{q_{S}}, y^{\prime}\right)\right)
$$

with

$$
F_{\mathcal{I}, S^{\prime}}\left(\left(x^{\prime}\right)^{q_{S}}, y^{\prime}\right)=\left(y^{\prime}+\mu\left(x^{\prime}\right)^{q_{S}}\right)^{b_{S}} \prod_{1 \leq i \leq n, \mu_{i} \neq \mu}\left(y^{\prime}-\left(\mu_{i}-\mu\right)\left(x^{\prime}\right)^{q_{S}}\right)^{\nu_{i}} .
$$

For $\mu_{i} \neq \mu$ we perform in the $(x, y)$-coordinates the Newton map $\sigma_{\left(1, q_{S}, \mu_{i}\right)}$ :

$$
x=x_{1}, \quad y=x_{1}^{q_{S}}\left(y_{1}+\mu_{i}\right),
$$

and in the $\left(x^{\prime}, y^{\prime}\right)$-coordinates the map $\sigma_{\left(1, q_{S}, \mu_{i}-\mu\right)}$ :

$$
x^{\prime}=x_{1}^{\prime}, \quad y^{\prime}=\left(x_{1}^{\prime}\right)^{q_{S}}\left(y_{1}^{\prime}+\mu_{i}-\mu\right) .
$$

A simple computation yields $x_{1}=x_{1}^{\prime}$ and $y_{1}=y_{1}^{\prime}$.

Now we replace the Newton map $\sigma_{\left(1, q_{S}, \mu\right)}$ by the change of variables

$$
x^{\prime}=x, \quad y^{\prime}=y-\mu x^{q_{S}},
$$

yielding

$$
x^{\prime}=x_{1}, \quad y^{\prime}=x_{1}^{q_{S}} y_{1} .
$$

Then $x_{1}^{\alpha} y_{1}^{\beta}=\left(x^{\prime}\right)^{\alpha-q_{S} \beta}\left(y^{\prime}\right)^{\beta}$. Let $\alpha^{\prime}=\alpha-q_{S} \beta, \beta^{\prime}=\beta$. This is a linear automorphism of the plane. Any face of the original second Newton polygon with equation $p \alpha+r \beta=N$ corresponds to a face on the new first Newton polygon with equation $p \alpha^{\prime}+\left(r+p q_{S}\right) \beta^{\prime}=N$, hence lying under $S^{\prime}$. Analogously, with the Newton map $\sigma_{\left(1, q_{S},-\mu\right)}$ :

$$
x^{\prime}=x_{1}^{\prime}, \quad y^{\prime}=\left(x_{1}^{\prime}\right)^{q_{S}}\left(y_{1}^{\prime}-\mu\right)
$$

one verifies that $x^{\alpha} y^{\beta}=\left(x_{1}^{\prime}\right)^{\alpha+q_{S} \beta}\left(y_{1}^{\prime}\right)^{\beta}$ and that a face under $S$ of the original first polygon corresponds to a face of the new second polygon. Consequently $\mathcal{T}^{\prime}$ is indeed the Newton tree of $\mathcal{I}^{\prime}$. 
If the horizontal edge we have chosen ends with an arrow, we have $\mathcal{I}=(y+h(x))^{\nu} \mathcal{I}_{1}$, with $h(x) \in x \mathbb{C}[[x]], h(x) \neq 0$ and $\nu>0$. In that case, we perform the change of variables $x^{\prime}=x, y^{\prime}=y+h(x)$.

Example 1 (continued). To obtain the minimal Newton tree in Figure 17, we first perform the change of coordinates

$$
x^{\prime}=x, \quad y^{\prime}=y+x .
$$

This leads to $\mathcal{I}^{\prime}=\left(\left(y^{\prime}-x^{\prime}\right)^{2}-3 x^{\prime}\right)\left(y^{\prime}\left(y^{\prime}-x^{\prime}\right)^{4},\left(y^{\prime}\right)^{3}+\left(x^{\prime}\right)^{8}\right)$. We can write $\left(y^{\prime}-x^{\prime}\right)^{2}-3 x^{\prime}$ as the product of a unit and a series of the form $x^{\prime}+h\left(y^{\prime}\right)$ with $h\left(y^{\prime}\right) \in y^{\prime} \mathbb{C}\left[\left[y^{\prime}\right]\right]$. After a second change of coordinates

$$
x^{\prime \prime}=x^{\prime}+h\left(y^{\prime}\right), \quad y^{\prime \prime}=y^{\prime}
$$

We obtain an ideal in $\mathbb{C}\left[\left[x^{\prime}, y "\right]\right]$ with the tree in Figure 17 as Newton tree.

Remark 4.6. Minimal Newton trees are not unique. For example, in Example 1 one can alternatively perform first the coordinate change

$$
x^{\prime}=-3 x+y^{2}, \quad y^{\prime}=y .
$$

After a second coordinate change

$$
x^{\prime \prime}=x^{\prime}, \quad y^{\prime \prime}=y^{\prime}-\frac{1}{3} x^{\prime}
$$

the resulting ideal has the Newton tree given in Figure 18.

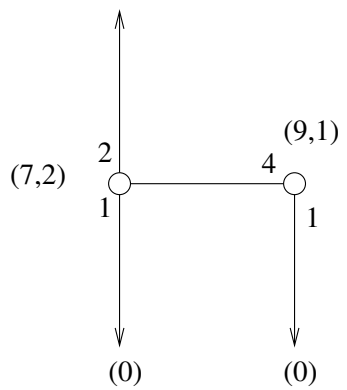

FiguRE 18.

Next we introduce the notion of (very) good coordinates; the point is that a Newton tree is minimal if and only if the Newton algorithm is performed in very good coordinates at each step.

Definition 4.7. Let $\mathcal{I}$ be a non-trivial ideal in $\mathbb{C}[[x, y]]$ and $\mathcal{N}(\mathcal{I})=\cup_{1 \leq i \leq m} S_{i}$ its Newton polygon. We say that $\mathcal{I}$ is in good coordinates if $\operatorname{in}\left(\mathcal{I}, S_{m}\right)$ is not a principal ideal generated by a polynomial of the form $x^{k}\left(y-\mu x^{q}\right)^{l}$ with $k \geq 0, l \geq 1$ and $\mu \in \mathbb{C}^{*}$.

Lemma 4.8. Let $\mathcal{I}$ be a non-trivial ideal in $\mathbb{C}[[x, y]]$. There exists a system of coordinates which is good for $\mathcal{I}$. 
Proof. Assume that the ideal $\mathcal{I}$ is not in good coordinates. Then there exist $k \geq 0, l \geq 1$ and $\mu \in \mathbb{C}^{*}$ such that

$$
\operatorname{in}\left(\mathcal{I}, S_{m}\right)=\left(x^{k}\left(y-\mu x^{q}\right)^{l}\right) .
$$

Consider the automorphism of $\mathbb{C}^{2}$ defined by $x=x^{\prime}, y=y^{\prime}+\mu\left(x^{\prime}\right)^{q}$. This automorphism does not change the faces $S_{1}, \cdots, S_{m-1}$ of $\mathcal{N}(\mathcal{I})$ but makes the face $S_{m}$ disappear. If this new system of coordinates is not good for $\mathcal{I}$, then we consider a new automorphism and so on. The process is finite if the ideal has $\{0\}$ as support. However, if the support of $\mathcal{I}$ contains a curve, that process can be infinite. In that case we use a coordinate change of the form $x=x^{\prime}, y=y^{\prime}+h\left(x^{\prime}\right)$ with $h\left(x^{\prime}\right) \in x^{\prime} \mathbb{C}\left[\left[x^{\prime}\right]\right]$. The details for this argument are the same as in the case of a principal ideal as explained in e.g. [13, Lemma 1.3] or [12, Theorem 6.40].

As an example of the first case, the ideal $\mathcal{I}_{1}=\left(y^{4}, x^{2}\left(x^{2}+y\right)+x^{3} y^{2}\right)$ is not in good coordinates. We obtain good coordinates after the change $x=x^{\prime}, y=y^{\prime}-\left(x^{\prime}\right)^{2}$. For the second case, consider $\mathcal{I}_{2}=\left(y+x^{2}+x y\right)$. Here the system of good coordinates is obtained by $x=x^{\prime}, y=y^{\prime}-\left(x^{\prime}\right)^{2}\left(1+x^{\prime}\right)^{-1}$.

Remark 4.9. Note that, in the procedure above, we do not eliminate dicritical faces.

Definition 4.10. Let $\mathcal{I}$ be a non-trivial ideal in $\mathbb{C}[[x, y]]$ and $\mathcal{N}(\mathcal{I})=\cup_{1 \leq i \leq m} S_{i}$ its Newton polygon. We say that $\mathcal{I}$ is in very good coordinates if the following conditions are satisfied.

- For $m>1$ : $\mathcal{I}$ is in good coordinates and $\operatorname{in}\left(\mathcal{I}, S_{1}\right)$ is not a principal ideal generated by a polynomial of the form $y^{k}\left(y^{p}-\mu x\right)^{l}$ with $k \geq 0, l \geq 1$ and $\mu \in \mathbb{C}^{*}$.

- For $m=1: \operatorname{in}\left(\mathcal{I}, S_{1}\right)$ is not a principal ideal generated by a polynomial of the form $x^{k}\left(y-\mu x^{q}\right)^{l}$ or $y^{k}\left(y^{p}-\mu x\right)^{l}$ as before, or $\left(y-\mu_{1} x\right)^{l_{1}}\left(y-\mu_{2} x\right)^{l_{2}}$ with $l_{1}, l_{2} \geq 1$ and $\mu_{1}, \mu_{2} \in \mathbb{C}^{*}$.

One verifies analogously that there exists a system of coordinates which is very good for $\mathcal{I}$. (For the last case one performs the coordinate change $y-\mu_{1} x=x^{\prime}, y-\mu_{2} x=y^{\prime}$.)

For example, the ideal $\mathcal{I}=\left(x^{2}-y^{2}, x^{3}\right)$ is not in very good coordinates. We obtain very good coordinates after the change $x=\left(x^{\prime}+y^{\prime}\right) / 2, y=\left(y^{\prime}-x^{\prime}\right) / 2$.

Remark 4.11. If $\mathcal{I}=\left(x^{N}\right) \mathcal{J}$ with $N \geq 1$, then good coordinates for $\mathcal{I}$ implies very good coordinates for $\mathcal{I}$.

The previous discussion shows the following.

Remark 4.12. The Newton tree is minimal if and only if the Newton algorithm is performed in very good coordinates at each step.

Remark 4.13. The notion of depth of an ideal from [7, see Definition 2.4, depends heavily on the chosen coordinates. In [7] we alluded to a possible more intrinsic notion. We can now define the geometric depth of an ideal in $\mathbb{C}[[x, y]]$ as the minimum of the widths of the minimal Newton trees of $\mathcal{I}$.

For example the ideal in Example 1 has depth 2 but geometric depth 1 . 


\section{GEOMETRIC INTERPRETATION}

In the case of a principal ideal $\mathcal{I}=(f)$ of $\mathbb{C}[[x, y]]$ it is shown in [6, Theorem 3.6] that the Newton tree of $\mathcal{I}$ is more or less equal to the dual graph of the minimal embedded resolution of $f$, where vertices of valence 2 are deleted. See also [13] (especially Lemma 2.12 and Theorem 3.1.2(i)). These proofs immediately generalize to arbitrary ideals, yielding the results in the theorem below.

We first recall the notion of (minimal) log principalization of an ideal (in our context).

Let $\mathcal{I}$ be a non-trivial ideal in $\mathbb{C}[[x, y]]$. A log principalization (or monomialization) of $\mathcal{I}$ is a proper birational morphism $h: X \rightarrow \operatorname{Spec} \mathbb{C}[[x, y]]$ from a smooth variety $X$ such that the ideal sheaf $\mathcal{I O}_{\bar{X}}$ is invertible and moreover its associated divisor of zeroes is a (strict) normal crossings divisor. The minimal $\log$ principalization of $\mathcal{I}$ is the unique such $h$, up to isomorphism, through which all other ones factorize.

Theorem 5.1. Let $\mathcal{I}$ be a non-trivial ideal in $\mathbb{C}[[x, y]]$ and $T$ a minimal Newton tree of $\mathcal{I}$. There exists a toroidal surface $\bar{X}$ and a proper birational morphism $\bar{\pi}: \bar{X} \rightarrow$ Spec $\mathbb{C}[[x, y]]$ with the following properties. Let $E_{j}, j \in J$, denote the irreducible components of the exceptional locus of $\bar{\pi}$ and $C_{i}, i \in I$, the strict transform of the one-dimensional irreducible components of the support of $\mathcal{I}$ (if any).

(1) There is a natural bijection between the $E_{j}$ and the vertices of $T$, and between the $C_{i}$ and the arrowheads of $T$ that are not decorated by $(0)$.

(2) The ideal sheaf $\mathcal{I O}_{\bar{X}}$ is invertible, with associated normal crossings divisor (in orbifold sense) $\bar{D}=\sum_{i \in I} N_{i} C_{i}+\sum_{j \in J} N_{j} E_{j}$, where the coefficients $N_{j}$ and $N_{i}$ are the decorations of the corresponding vertices and arrows, respectively, of $T$.

(3) The singularities of $\bar{X}$ are located on the intersections of the components $E_{j}$. They are cyclic quotient singularities of order equal to the edge determinant of the corresponding edge in $T$.

The composition of the resolution map $h: X \rightarrow \bar{X}$ of these singularities and the map $\bar{\pi}$ yields the minimal log principalization map $\pi: X \rightarrow \operatorname{Spec} \mathbb{C}[[x, y]]$ of the ideal $\mathcal{I}$.

Proof. The argument for principal ideals can be found in [6, §3], and is identical for arbitrary ideals. Indeed, the proof of Theorem 3.6 in [6] only uses the interpretation of the Newton tree of a germ as representing successive Newton polygons and Newton maps, as does the Newton tree of an ideal. Then it proves also Theorem 5.1 .

Morally the tree $T$ is the dual graph of the divisor $\bar{D}$ in (2). More precisely, one obtains this dual graph by erasing in $T$ the arrowheads decorated with (0) and all edges starting from those arrowheads and decorated by 1 on the other side.

What was still missing is the meaning of the edge decorations of $T$ in the dual graph of $\bar{D}$. The next proposition says that these edge decorations have the 'correct' interpretation as classical edge decorations in a dual graph.

Proposition 5.2. We use the notation of Theorem 5.1. Denote $D=h^{*} \bar{D}$. The decoration on an edge e near a vertex $v$ of the Newton tree $T$ is the absolute value of the determinant of the intersection matrix of those components of $D$ (in the surface $X$ ), corresponding to the part of the tree away from $v$ in the direction of $e$.

Proof. We first fix some terminology. We will often talk about the determinant of minus the intersection matrix (which is the same as the absolute value of the determinant of the intersection matrix) of a configuration $C$ of (irreducible) curves on a smooth surface. For sake of brevity we denote $\operatorname{det}(C)$ for this determinant. 
Recall that $\operatorname{det}(\cdot)$ is invariant under blowing up: when $C$ is a configuration of curves on a smooth surface $S$ and $\tilde{S} \rightarrow S$ is a point blow-up with exceptional curve $E$, then, denoting by $\tilde{C}$ the configuration in $\tilde{S}$ of strict transforms of curves in $C$, we have $\operatorname{det}(C)=\operatorname{det}(\tilde{C} \cup\{E\})$.

Also, with the configuration $C$ of curves associated to a part $\Delta$ of the Newton tree $T$ we mean the total transform in $X$ of the configuration of curves in $\bar{X}$, associated by the bijection in Theorem 5.1 (1) to the vertices in $\Delta$. We then $\operatorname{define} \operatorname{det}(\Delta):=\operatorname{det}(C)$. With this terminology we must show:

any decoration on an edge e near a vertex $v$ of $T$ is equal to $\operatorname{det}(\Delta)$, where $\Delta$ is the connected component of $T \backslash\{v\}$ in the direction of $e$.

(i) Any (implicit) edge decoration 1 on the left of a horizontal edge (i.e., right to a vertex) satisfies $(*)$.

Indeed, the associated part $\Delta$ corresponds in $X$ to the total exceptional locus of a composition of blow-ups.

(ii) Any edge decoration on the first vertical line satisfies $(*)$.

Fix a vertex $v$ on the first vertical line of $T$ with edge decorations $q$ and $p$ above and below $v$, respectively. Denote by $Q_{1}$ and $P_{1}$ the parts of $T$ on the first vertical line above and below $v$, respectively, and by $Q$ and $P$ the total connected components of $T \backslash\{v\}$ in the direction of $q$ and $p$, respectively. E.g. [5, Theorem 10.4.4] immediately yields $q=\operatorname{det}\left(Q_{1}\right)$ and $p=\operatorname{det}\left(P_{1}\right)$. And because $\operatorname{det}(\cdot)$ is invariant under blowing up, we have $\operatorname{det}\left(Q_{1}\right)=\operatorname{det}(Q)$ and $\operatorname{det}\left(P_{1}\right)=\operatorname{det}(P)$. We conclude that $q=\operatorname{det}(Q)$ and $p=\operatorname{det}(P)$, finishing case (ii).

(iii) Any edge decoration below a vertex on another vertical line satisfies $(*)$.

Because the edge decorations below vertices do not change during the construction of the Newton tree $T$, we can use exactly the same arguments as in the previous case.

(iv) Any edge decoration above (or left to) a vertex on another vertical line satisfies (*).

Here we use induction on the number of preceding vertices of the given vertex. Fix a vertex $v$ with edge decorations $q$ and $p$, and say $v_{0}$, with edge decorations $q_{0}$ and $p_{0}$, is its preceding vertex (see Figure 19).

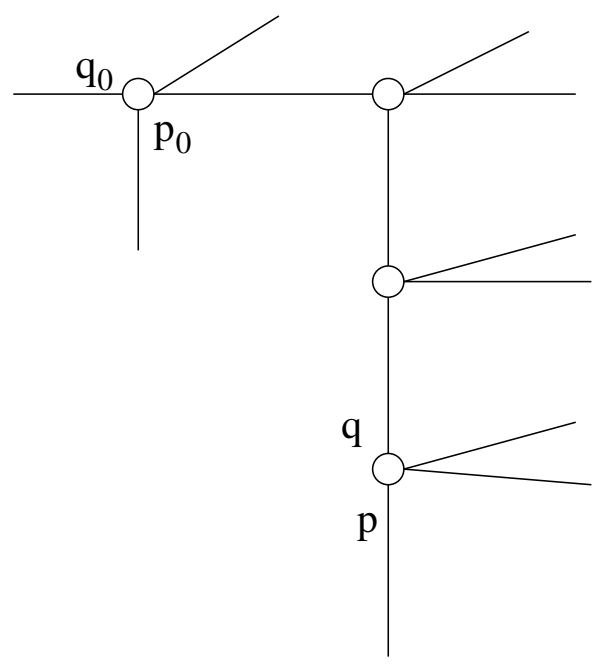

FIGURE 19. 
Let also $m$ be the original decoration above $v$ when drawing the vertical line of $v$ associated to a Newton diagram. Then by [7, Proposition 3.1] we have

$$
q=p_{0} q_{0} p+m \text {. }
$$

We introduce notation for the relevant parts of $T$. Let $Q$ and $P$ be the connected components of $T \backslash\{v\}$ in the direction of $q$ and $p$, respectively, and similarly $Q_{0}$ and $P_{0}$. Let also $\Delta$ denote the chain between $v$ and $v_{0}$, i.e., the part above $v$ of the original vertical line of $v$ associated to a Newton diagram. As in (ii), we have that

$$
m=\operatorname{det}(\Delta), \quad p=\operatorname{det}(P) \quad \text { and } \quad p_{0}=\operatorname{det}\left(P_{0}\right) .
$$

Our induction hypothesis says that

$$
q_{0}=\operatorname{det}\left(Q_{0}\right)
$$

Combining (5.1), (5.2) and (5.3) yields $q=\operatorname{det}\left(P_{0}\right) \operatorname{det}\left(Q_{0}\right) \operatorname{det}(P)+\operatorname{det}(\Delta)$. Finally by a (well known) determinant computation this right hand side is equal to $\operatorname{det}(Q)$.

Under the bijective correspondence in Theorem 5.1 between vertices of $T$ and exceptional components of $\bar{\pi}$, the dicritical vertices of $T$ have a conceptual geometric meaning. We fix some notation in order to describe this relation.

Let $b: B \rightarrow \operatorname{Spec} \mathbb{C}[[x, y]]$ denote the normalized blow-up of the ideal $\mathcal{I}$. Since the ideal sheaf $\mathcal{I O}_{\bar{X}}$ is invertible, the map $\bar{\pi}: \bar{X} \rightarrow \operatorname{Spec} \mathbb{C}[[x, y]]$ factorizes through $b$, yielding a morphism $p: \bar{X} \rightarrow B$.

Theorem 5.3. We use the notation of Theorem 5.1. Under the bijection (1), the dicritical vertices of the Newton tree $T$ correspond with the exceptional components of the normalized blow-up of the ideal $\mathcal{I}$, that $i s$, with those exceptional components of $\bar{\pi}$ that are mapped onto a curve by $p$.

Proof. By [7, Proposition 5.12] the dicritical vertices of $T$ correspond to the Rees valuations of $\mathcal{I}$. Next use [16].

In fact the morphism $p: \bar{X} \rightarrow B$ is a natural and important 'partial resolution' of the divisor of $\mathcal{I O}_{B}$ in $B$ in the framework of the Minimal Model Program; it is precisely the relative $\log$ canonical model of this divisor in $B$. We refer to [11] for definitions.

Proposition 5.4. Let $\mathcal{I}$ and $\omega$ be as in Definition 3.2.

(i) The motivic zeta function $\zeta(\mathcal{I}, \omega)(T)$ only depends on a minimal Newton tree of the ideal.

(ii) When performing the Newton algorithm in very good coordinates, all candidate poles $\mathbb{L}^{s_{0}}\left(s_{0} \in \mathbb{Q}\right)$ in the formula of Theorem 3.4 for $\zeta(\mathcal{I}, d x \wedge d y)(T)$ are true poles.

Proof. (i) This is clear.

(ii) This follows from [17] and the geometric interpretation of the Newton tree; we sketch the argument. Theorem 4.1 in [17] describes the poles of $\zeta(\mathcal{I}, d x \wedge d y)(T)$ in terms of the minimal principalization of $\mathcal{I}$. (In fact, the result is stated there for the topological zeta function, but is clearly also valid for the motivic zeta function, see [17, §6].) More adequate for the link with the present paper, is the following reformulation of this description as in [18, Theorem 4.4]. We use the notation of Theorem 5.1, we moreover denote the exceptional components of $h$ by $E_{j}, j \in J^{\prime}$, and we put $D=\widehat{h^{*} X}=\sum_{i \in I} N_{i} C_{i}+\sum_{j \in J \cup J^{\prime}} N_{j} E_{j}$ and $\pi^{*}(d x \wedge d y)=\sum_{j \in J \cup J^{\prime}}\left(n_{j}-1\right) E_{j}$.

Then $\mathbb{L}^{s_{0}}$ is a pole of $\zeta(\mathcal{I}, d x \wedge d y)(T)$ if and only if

(1) $s_{0}=\frac{1}{N_{i}}$ for some $i \in I$; 
(2) $s_{0}=\frac{n_{j}}{N_{j}}$ for some $j \in J \cup J^{\prime}$ such that $E_{j}$ intersects at least three times other components $E_{\ell}$ or $C_{i}$;

(3) $s_{0}=\frac{n_{j}}{N_{j}}$ for some $j \in J \cup J^{\prime}$ such that $E_{j}$ has non-empty intersection with the strict transform of a generic curve of the ideal $\mathcal{I}$.

Note that then in fact $j \in J$, since the $E_{j}, j \in J^{\prime}$, intersect at most two other components and do not intersect the strict transform of a generic curve (since they are contracted by $h$ ).

These $\mathbb{L}^{s_{0}}$ are precisely all the candidate poles in the formula of Theorem 3.4 for $\zeta(\mathcal{I}, d x \wedge$ $d y)(T)$. Indeed, case (1) corresponds to the arrowheads with nonzero decoration in the minimal Newton tree/dual graph $T$, and cases (2) and (3) correspond to the vertices in $T$. More precisely, according to Theorem 5.3. case (3) corresponds to the dicritical vertices of $T$, since intersecting the strict transform of a generic curve is equivalent to being an exceptional component of the normalized blow-up. (The numbers $n_{j}$ are really the numbers of the form $p_{\ell} \nu+q_{\ell}$ in the formula of Theorem 3.4 , see $\S 6$ below.)

\section{THE LOG CANONICAL THRESHOLD}

The log canonical threshold is an important singularity invariant. We refer to [14] for various equivalent definitions and more information. We only mention the description that we need, for ideals in $\mathbb{C}[[x, y]]$, using the notation of Theorem 5.1 .

Recall that $\pi: X \rightarrow \operatorname{Spec} \mathbb{C}[[x, y]]$ is the minimal log principalization of $\mathcal{I}$. We denote the irreducible components of $D=\mathcal{I O}_{X}$ by $F_{\ell}, \ell \in L$; they consist of the exceptional components of $h$ and (the strict transforms of) the $E_{j}$ and $C_{i}$. Write $D=\sum_{\ell \in L} N_{\ell} F_{\ell}$ and $\pi^{*}(d x \wedge d y)=$ $\sum_{\ell \in L}\left(n_{\ell}-1\right) E_{\ell}$.

Definition 6.1. Let $\mathcal{I}$ be a non-trivial ideal in $\mathbb{C}[[x, y]]$. The log canonical threshold lct $(\mathcal{I})$ of $\mathcal{I}$ is the minimum of the numbers $\frac{n_{\ell}}{N_{\ell}}, \ell \in L$.

Remark 6.2. The minimum above is in fact always obtained on components of $\bar{D}$, that is, $l c t(\mathcal{I})$ is the minimum of the numbers $\frac{n_{\ell}}{N_{\ell}}, \ell \in I \cup J$. See e.g. [17, Section 3].

Under the correspondence in Theorem 5.1 one can also describe the $n_{\ell}$ in terms of the Newton tree $T$. Recall that a component of $D$ corresponds to a vertex or arrowhead in the tree, hence also to a face of some Newton diagram appearing in the Newton algorithm of $\mathcal{I}$.

We will use the following terminology: after a composition of Newton maps, we call the pullback of $d x \wedge d y$ the differential form at that stage of the Newton algorithm.

Lemma 6.3. Let $\mathcal{I}$ be a non-trivial ideal in $\mathbb{C}[[x, y]]$. Let $S$ be a face, with equation $p_{S} \alpha+$ $q_{S} \beta=N_{S}$, of some Newton diagram appearing in the Newton algorithm of $\mathcal{I}$. Let $F_{S}$ be the component of $D$ corresponding to $S$. Then $n_{S}=p_{S} \nu+q_{S}$, where $x^{\nu-1} d x \wedge d y$ is the differential form at that stage of the Newton algorithm (in local coordinates $x, y$ ).

Proof. This follows immediately from [6, Proposition 3.9].

Proposition 6.4. The log canonical threshold of a non-trivial ideal $\mathcal{I}$ in $\mathbb{C}[[x, y]]$ is the minimum of the numbers $\frac{p_{S} \nu+q_{S}}{N_{S}}$, where $S$ runs over all faces, with equation $p_{S} \alpha+q_{S} \beta=N_{S}$, of all the Newton diagrams appearing in the Newton algorithm of $\mathcal{I}$, and where $x^{\nu-1} d x \wedge d y$ is the differential form at that stage of the Newton algorithm.

Proof. Combine Remark 6.2 and Lemma 6.3. 
Theorem 6.5. Let $\mathcal{I}$ be a non-trivial ideal in $\mathbb{C}[[x, y]]$. Then there exists a change of coordinates $\phi:(x, y) \mapsto\left(x^{\prime}, y^{\prime}\right)$ such that $l \operatorname{ct}(\mathcal{I})=\frac{p+q}{N}$ where $S$, with equation $p \alpha+q \beta=N$, is the face of the first Newton diagram of the ideal $\mathcal{I}^{\prime}=\phi(\mathcal{I})$ in $\mathbb{C}\left[\left[x^{\prime}, y^{\prime}\right]\right]$, intersected by the line $\alpha=\beta$.

To prove the theorem, we need a series of lemmas. The first one is quite obvious.

Lemma 6.6. Let $\mathcal{N}$ be a Newton diagram whose faces have equation $p_{i} \alpha+q_{i} \beta=N_{i}$ with $\left(p_{i}, q_{i}\right)=1$ for $i=0, \cdots, m+1$ and $p_{0}=1, q_{0}=0, p_{m+1}=0, q_{m+1}=1$. Let $\nu$ be a positive integer. Then $\min _{0 \leq i \leq m+1} \frac{p_{i} \nu+q_{i}}{N_{i}}=\frac{1}{t}$, where $t$ is the ordinate of the point of intersection of the line $\alpha=\nu \beta$ with the Newton diagram.

Corollary 6.7. The minimum of the values $\frac{p \nu+q}{N}$ on the first Newton diagram is obtained by $1 / t$, where $t$ is the ordinate of the point where the line $\alpha=\nu \beta$ intersects the Newton diagram.

Definition 6.8. Let $S$ be a face, with equation $p \alpha+q \beta=N$, of a Newton diagram appearing in the Newton algorithm, with differential $w=x^{\nu-1} d x \wedge d y$ at that stage. The rational number $\frac{n}{N}=\frac{p \nu+q}{N}$ is called the invariant of $S$.

We are looking for the smallest invariant appearing in the Newton process.

Corollary 6.9. Let $S_{0}$ be a face of a Newton polygon where the invariant is $\frac{n_{0}}{N_{0}}$. Let $\mu_{0}$ be a root of its face polynomial with multiplicity $m_{0}$. Assume $m_{0} n_{0}<N_{0}$. Consider the Newton map associated to $S_{0}$ and $\mu_{0}$. Then for all faces of the new Newton diagram, the invariant $\frac{n}{N}$ satisfies $\frac{n}{N} \geq \frac{n_{0}}{N_{0}}$.

Proof. After applying the Newton map $\sigma_{\left(p, q, \mu_{0}\right)}$ associated to $S_{0}$ and $\mu_{0}$, we have a Newton diagram with a non-compact face $\alpha=N_{0}$, and the highest point on the Newton polygon has coordinates $\left(N_{0}, m_{0}\right)$. The hypothesis $m_{0} n_{0}<N_{0}$ says that the line $\alpha=n_{0} \beta$ intersects the face $\alpha=N_{0}$ (see Figure 20). Then the minimum of the values $\frac{p m_{0}+q}{N}$ on all the faces of the new diagram is $\frac{n_{0}}{N_{0}}$. But the differential at this stage of the Newton process is $w=x^{n_{0}-1} d x \wedge d y$.

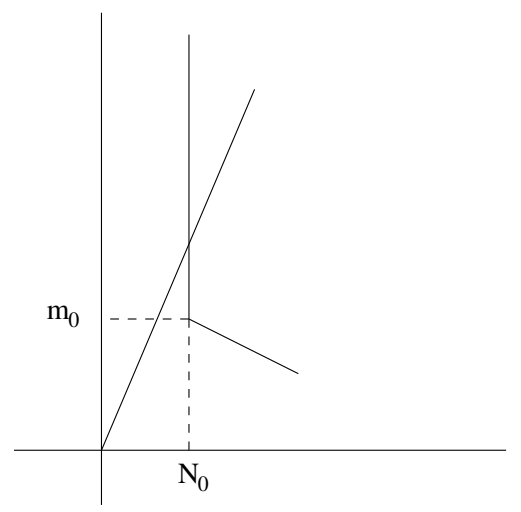

FigURE 20.

Lemma 6.10. Let $S_{0}$ be a face of a Newton polygon where the invariant is $\frac{n_{0}}{N_{0}}$. Let $\mu_{0}$ be a root of its face polynomial with multiplicity $m_{0}$. Consider the Newton map associated to $S_{0}$ and $\mu_{0}$. Let $S$ be a face of the new Newton polygon with invariant $\frac{n}{N}$, and let $\mu$ be a root of its face polynomial with multiplicity $m$. If $n_{0} m_{0}<N_{0}$, then $n m<N$. 
Proof. Write the equation of $S$ as $p \alpha+q \beta=N$, and let $m^{\prime}$ be the ordinate of the point where $S$ intersects the line $\alpha=N_{0}$ (Figure 21). We have $p N_{0}+q m^{\prime}=N$ and

Then

$$
m \leq m^{\prime} \leq m_{0}<\frac{N_{0}}{n_{0}}
$$

$$
m n-N=m\left(p n_{0}+q\right)-N \leq p m_{0} n_{0}+m^{\prime} q-N<p N_{0}+q m^{\prime}-N=0
$$

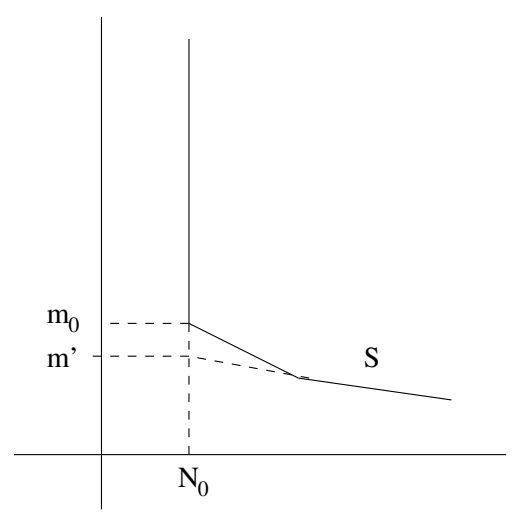

FIGURE 21.

Next we study the case where $n_{0} m_{0} \geq N_{0}$.

Lemma 6.11. Let $S_{0}$ be a face of a Newton polygon with equation $p_{0} \alpha+q_{0} \beta=N_{0}$ and with invariant $\frac{p_{0}+q_{0}}{N_{0}}$. Let $\mu_{0}$ be a root of its face polynomial with multiplicity $m_{0}$. If $n_{0} m_{0} \geq N_{0}$, then $p_{0}$ or $q_{0}$ is equal to 1.

Proof. We associate to the initial ideal with respect to $S_{0}$ the numbers $a, b$ and $d$ as in (2.1) or (2.2), and we denote by $m_{\mu}$ de multiplicity of another root $\mu$ of the face polynomial of $S_{0}$. Then we have

$$
N_{0}=a p_{0}+b q_{0}+p_{0} q_{0}\left(m_{0}+\sum_{\mu \neq \mu_{0}} m_{\mu}+d\right) .
$$

Hence

$$
0 \leq n_{0} m_{0}-N_{0}=\left(p_{0}+q_{0}\right) m_{0}-N_{0} \leq\left(p_{0}+q_{0}\right) m_{0}-p_{0} q_{0} m_{0}
$$

And $p_{0}+q_{0}-p_{0} q_{0} \geq 0$ implies that $p_{0}$ or $q_{0}$ is equal to 1 (since $p_{0}$ and $q_{0}$ are coprime).

Now we can prove Theorem 6.5.

Proof. We fix a vertex (or arrow) $v$ in the Newton tree of $\mathcal{I}$ where the minimum value of the invariant $\frac{n}{N}$ is attained, such that no preceding vertex of $v$ has that minimum as invariant.

If $v$ corresponds to a face on the first Newton polygon, we know that the minimum is given by the intersection with the line $\alpha=\beta$. If it is not on the first vertical line of the Newton tree, then we consider the sequence of vertices which lead from the first vertical line to $v$. Let $v_{0}$ be the vertex of that sequence on the first vertical line. We claim that $n_{0} m_{0} \geq N_{0}$. Indeed, otherwise Lemma 6.10 and Corollary 6.9 provide a contradiction.

Hence by Lemma 6.11 we have that $p_{0}$ or $q_{0}$ is equal to 1 . Say $p_{0}=1$. Let $v_{1}$ be the second vertex of the sequence. We can perform the exchange of vertical edge between the edge decorated by $p_{0}=1$ and the horizontal edge with extremities $v_{0}$ and $v_{1}$. If $v_{1}=v$, then $v$ is now on the first Newton polygon and as its invariant is the minimum, it is given by the 
intersection with the line $\alpha=\beta$. If $v_{1} \neq v$, we can conclude similarly that $n_{1} m_{1} \geq N_{1}$, apply Lemma 6.11 to $v_{1}$ and perform an exchange of vertical edge. We continue until $v$ is on the first vertical line. As we showed that an exchange of vertical edge corresponds to a change of coordinates, we are done.

Remark 6.12. Even in very good coordinates, the log canonical threshold may appear at the second (or later) step of the Newton algorithm. Here is an example:

$$
\mathcal{I}=\left((y+x)^{5}, x^{2}(y+x)^{3}, x^{8}\right) .
$$

In these coordinates, the Newton tree is as in Figure 22. One easily verifies that $l c t(\mathcal{I})=\frac{3}{8}$, appearing at the second step.

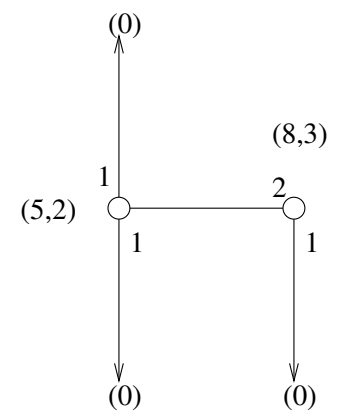

Figure 22.

Remark 6.13. We should note that the log canonical threshold of the generic curve of an ideal may be different from the log canonical threshold of the ideal. For example, the log canonical threshold of the ideal $\mathcal{I}=(x, y)$ is 2 , but the log canonical threshold of the curve $\{x+a y=0\}$ is 1 .

The next results of [8] and [4] are immediate consequences of the previous theorem. Let $\mathcal{M}$ denote the maximal ideal of $\mathbb{C}[[x, y]]$. Also, for an ideal $\mathcal{I}$ in $\mathbb{C}[[x, y]]$ of finite codimension, we denote by $m(\mathcal{I})$ the area delimited by the axes and the Newton polygon of $\mathcal{I}$.

Corollary 6.14. Assume that $\mathcal{I}$ is an ideal in $\mathbb{C}[[x, y]]$ of finite codimension. Let e $(\mathcal{I})$ be its Hilbert-Samuel multiplicity.

(1) Then

$$
e(\mathcal{I}) \geq \frac{4}{\operatorname{lct}(\mathcal{I})^{2}}
$$

(2) We have equality if and only if there is a positive integer $N$ such that the integral closure of $\mathcal{I}$ is equal to $\mathcal{M}^{N}$. Moreover, in this case $N=\frac{2}{\operatorname{lct}(\mathcal{I})}$.

Proof. (1) We have proven that there is an ideal $\mathcal{I}^{\prime}$ isomorphic to $\mathcal{I}$ such that the log canonical threshold of $\mathcal{I}^{\prime}$ is the ordinate of the intersection of the line $\alpha=\beta$ with the Newton polygon of $\mathcal{I}^{\prime}$. Then the Hilbert-Samuel multiplicity of $\mathcal{I}^{\prime}$ is the same as the Hilbert-Samuel multiplicity of $\mathcal{I}$. Hence we can assume that $\mathcal{I}=\mathcal{I}^{\prime}$. It is known [7] that $e(\mathcal{I}) \geq 2 m(\mathcal{I})$. One easily verifies that $2 \frac{1}{\operatorname{lct}(\mathcal{I})^{2}} \leq m(\mathcal{I})$.

(2) We just derived that equality occurs if and only if

$$
e(\mathcal{I})=2 m(\mathcal{I})=\frac{4}{\operatorname{lct}(\mathcal{I})^{2}}
$$


The first equality occurs if and only if $\mathcal{I}$ is non-degenerate, the second one if and only if the Newton polygon of $\mathcal{I}$ has exactly one compact face, with equation of the form $\alpha+\beta=N$. Taking into account the results of [7] these statements mean that the integral closure of $\mathcal{I}$ is a power of $\mathcal{M}$.

We can give a stronger inequality using the exact computation of the Hilbert-Samuel multiplicity of an ideal [7]. There we computed $e(\mathcal{I})$ using the area of the regions associated with the successive Newton polygons that appear in the Newton algorithm. If $\Sigma_{i}=\left(\sigma_{1}, \cdots, \sigma_{i}\right)$ is a sequence of Newton maps, then $\mathcal{I}_{\Sigma_{i}}$ is of the form $\mathcal{I}_{\Sigma_{i}}=x^{N_{i}} \mathcal{I}_{\Sigma_{i}}^{\prime}$, where $\mathcal{I}_{\Sigma_{i}}^{\prime}$ is of finite codimension

Theorem 6.15. [7, Theorem 5.18] Let $\mathcal{I}$ be a non-trivial ideal in $\mathbb{C}[[x, y]]$ of finite codimension and of depth $d$. Then

$$
e(\mathcal{I})=2\left(m(\mathcal{I})+\sum_{i=1}^{d} \sum_{\Sigma_{i}} m\left(\mathcal{I}_{\Sigma_{i}}^{\prime}\right)\right),
$$

the second summation being taken over all possible sequences of Newton maps of length $i$.

Theorem 6.16. Let $\mathcal{I}$ be a non-trivial ideal in $\mathbb{C}[[x, y]]$ of finite codimension. Consider a system of coordinates such that the log canonical threshold is given by its Newton diagram. Then

$$
e(\mathcal{I}) \geq 4\left(\frac{1}{l c t(\mathcal{I})^{2}}+\sum_{\sigma} \frac{1}{l c t\left(\mathcal{I}_{\sigma}^{\prime}\right)^{2}}\right)
$$

where the summation is taken over all Newton maps associated to the faces of the Newton diagram and the roots of the face polynomials.

Proof. Theorem 6.15 can be rewritten as

$$
e(\mathcal{I})=2 m(\mathcal{I})+\sum_{\sigma} e\left(\mathcal{I}_{\sigma}^{\prime}\right)
$$

where the summation is taken over all Newton maps associated to the faces of the Newton diagram of $\mathcal{I}$ and the roots of the face polynomials. If moreover we choose a system of coordinates such that the log canonical threshold is given by the Newton polygon, we have $2 m(\mathcal{I}) \geq 4 \frac{1}{\operatorname{lct}(\mathcal{I})^{2}}$. Using Corollary 6.14, we get the result.

As an example, we take Example 3 in [7]. We consider in $\mathbb{C}[[x, y]]$ the ideal

$$
\mathcal{I}=\left(y^{2}\left(\left(x^{2}+y^{3}\right)^{2}+x y^{5}\right)\left(x^{2}-y^{3}\right), x^{8} y+x^{12}\right) .
$$

We computed in [7] that $e(\mathcal{I})=102$. There are two possible minimal Newton trees, drawn in Figure 23.

In both cases the log canonical threshold $\frac{5}{22}$ is computed from the Newton polygon. Corollary 6.14 applied to this example gives $102>77,44$. Theorem 6.16 gives with the system of coordinates which gives the Newton tree on the left hand side $102>85,72$, and if we use coordinates on the right hand side $102>83.16$. 

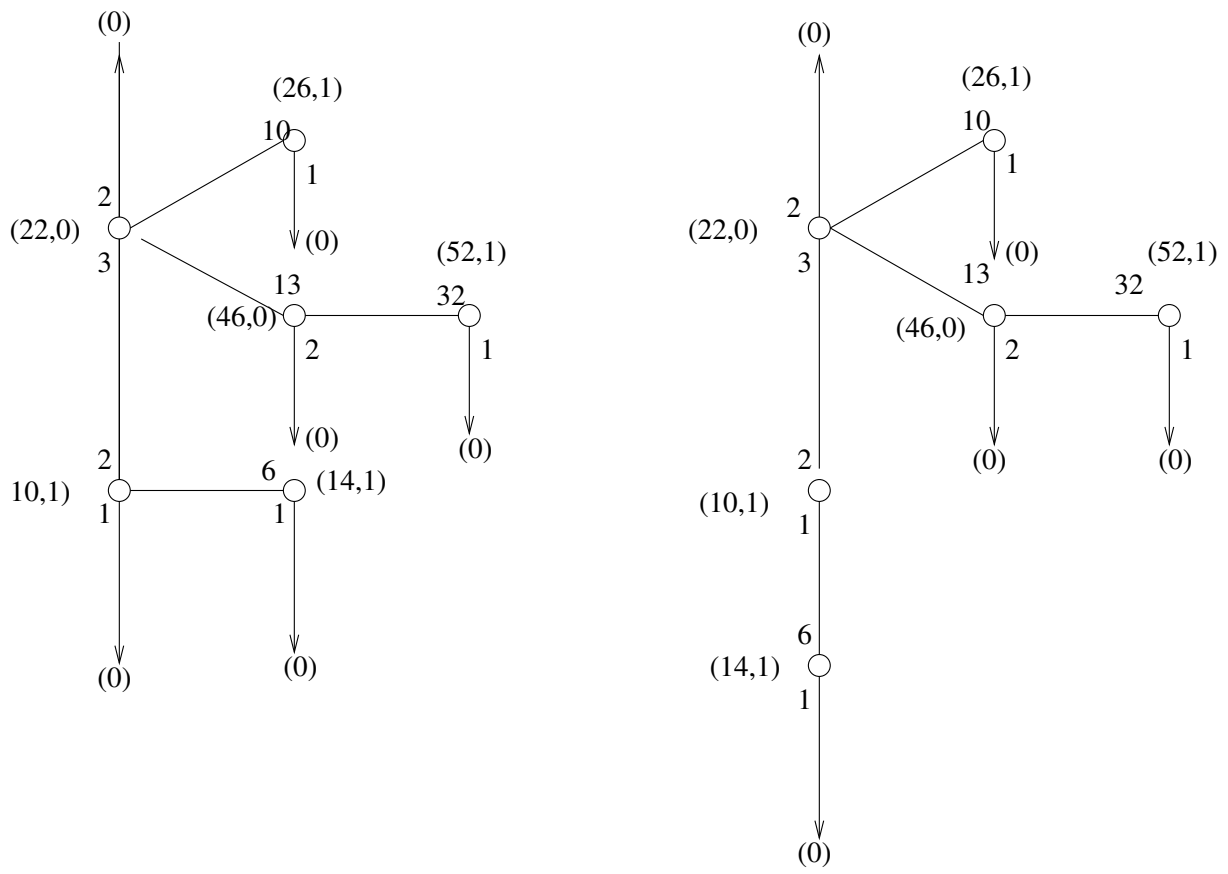

FiguRE 23.

\section{REFERENCES}

[1] E. Artal, P. Cassou-Noguès, I. Luengo and A. Melle Hernández Quasi-ordinary power series and their zeta functions, Mem. Amer. Math. Soc. 178 (2005), vi +85.

[2] E. Artal, P. Cassou-Noguès, I. Luengo and A. Melle Hernández $\nu$-Quasi-ordinary power series: factorisation, Newton trees and resultants, Topology of algebraic verieties and singularities, Contemp. Math. 538 (2011), 321-343.

[3] E. Artal, P. Cassou-Noguès, I. Luengo and A. Melle Hernández On the log-canonical threshold for germs of plane curves, Singularities I, Contemp. Math. 474 (2008), 1-14.

[4] A. CorTi Singularities of linear systems and 3-fold birational geometry, in Explicit birational geometry of 3-folds, 259-312, Cambridge Univ. Press, Cambridge, 2000.

[5] D. Cox, J. Little And H. Schenck Toric varieties, Graduate Studies in Mathematics 124, American Mathematical Society, Providence, RI, 2011.

[6] P. Cassou-Noguìs and A. Libgober Multivariate Hodge theorical invariants of germs of plane curves. II, The Valuation Theory Home Page, Preprint 228.

[7] P. Cassou-Noguès and W. Veys Newton trees for ideals in two variables and applications, Proc. London Math. Soc. doi:10.1112/plms/pdt047 (2013), 42p.

[8] T. De Fernex, L. Ein and M. Mustaţă Multiplicities and log canonical thresholds, J. Alg. Geom. 13 (2004), 603-615.

[9] J. Denef and F. Loeser Motivic Igusa zeta functions, J. Alg. Geom. 7 (1998), 505-537.

[10] J. Kollar Singularities of pairs, Algebraic Geometry-Santa Cruz 1995, Proc. Sympos. Pure Math., 62, Part 1, 221-287

[11] J. Kollár and S. Mori Birational geometry of algebraic varieties, Cambridge Tracts in Mathematics 134, Cambridge University Press, 2008.

[12] J. Kollár, K. Smith and A. Corti Rational and nearly rational varieties, Cambridge Studies in Advanced Mathematics 92, Cambridge University Press, Cambridge, 2004.

[13] D.T. LÊ AND M. OKa On resolution complexity of plane curves, Kodai Math. J. 18 (1996), 1-36.

[14] M. Mustaţă IMPANGA lecture notes on log canonical thresholds, Notes by Tomasz Szemberg, EMS Ser. Congr. Rep., Contributions to algebraic geometry, 407-442, Eur. Math. Soc., Zürich, 2012.

[15] J. Nicaise And J. Sebag Greenberg approximation and the geometry of arc spaces, Comm. Alg. 38 (2010), 4077-4096.

[16] I. Swanson and C. Huneke Integral closure of ideals, rings, and modules, Lond. Math. Soc. L.N.S. 336 (2006). 
[17] L. Van Proeyen and W. Veys Poles of the topological zeta function associated to an ideal in dimension two, Math. Z. 260 (2008), 615-627.

[18] L. Van Proeyen and W. Veys The monodromy conjecture for zeta functions associated to ideals in dimension two, Ann. Inst. Fourier 60 (2010), 1347-1362.

[19] W. Veys Zeta functions for curves and log canonical models, Proc. London Math. Soc. 74 (1997), 360-378.

[20] W. VEYs Zeta functions and 'Kontsevich Invariants' on singular varieties, Canad. J. Math. 53 (2001), 834-865.

[21] W. VEYs AND W. ZÚÑIGA-GaLindo Zeta functions for analytic mappings, log-principalization of ideals, and Newton polyhedra, Trans. Amer. Math. Soc. 360 (2008), 2205-2227.

Institut de Mathématiques de Bordeaux, Université Bordeaux I, 350, Cours de la LibéraTion, 33405, Talence Cedex 05, FRANCE

Ku Leuven, Dept. Wiskunde, Celestijnenlaan 200B, 3001 Leuven, Belgium 